\title{
THE
}

6-14-2006

\section{Gravitational Quantum States of Neutrons in a Rough Waveguide}

\author{
A. E. Meyerovich \\ University of Rhode Island, sfo101@uri.edu
}

V. V. Nesvizhevsky

Follow this and additional works at: https://digitalcommons.uri.edu/phys_facpubs

Terms of Use

All rights reserved under copyright.

\section{Citation/Publisher Attribution}

Meyerovich, A. E., \& Nesvizhevsky, V. (2006). Gravitational Quantum States of Neutrons in a Rough Waveguide. Phys. Rev. A, 73(6), 063616. doi: 10.1103/PhysRevA.73.063616

Available at: http://dx.doi.org/10.1103/PhysRevA.73.063616

This Article is brought to you for free and open access by the Physics at DigitalCommons@URI. It has been accepted for inclusion in Physics Faculty Publications by an authorized administrator of DigitalCommons@URI. For more information, please contact digitalcommons-group@uri.edu. 


\title{
Gravitational quantum states of neutrons in a rough waveguide
}

\author{
A. E. Meyerovich \\ Department of Physics, University of Rhode Island, Kingston, Rhode Island 02881-0817, USA \\ V. V. Nesvizhevsky \\ ILL, Grenoble, France
}

(Received 20 March 2006; published 14 June 2006)

\begin{abstract}
A theory of gravitational quantum states of ultracold neutrons in waveguides with absorbing/scattering walls is presented. The theory covers recent experiments in which the ultracold neutrons were beamed between a mirror and a rough scatterer/absorber. The analysis is based on a recently developed theory of quantum transport along random rough walls which is modified in order to include leaky (absorbing) interfaces and, more importantly, the low-amplitude high-aperture roughness. The calculations are focused on a regime where the direct transitions into the continuous spectrum above the absorption threshold dominate the depletion of neutrons from the gravitational states and are more efficient than the processes involving the intermediate states. The theoretical results for the neutron count are sensitive to the correlation radius (lateral size) of surface inhomogeneities and to the ratio of the particle energy to the absorption threshold in the weak-roughness limit. The main impediment for observation of the higher gravitational states is the "overhang" of the particle wave functions which can be overcome only by using scatterers with strong roughness. In general, strong roughness with high amplitude is preferable if one wants just to detect the individual gravitational states, while strongroughness experiments with small amplitude and high aperture are preferable for the quantitative analysis of the data. We also discuss ways to further improve the accuracy of calculations and to optimize the experimental regime.
\end{abstract}

DOI: 10.1103/PhysRevA.73.063616

\section{INTRODUCTION}

One of the recent discoveries in neutron physics was the experimental observation of quantization of motion of ultracold neutrons in a gravitational field [1]. By itself, the quantization of motion of particles by a linear potential is a wellknown textbook problem in quantum mechanics and has already been observed for other ultracold particles (for example, for spin-polarized atomic hydrogen in a magnetic field with a linear gradient [2]). However, the Earth gravitational field is so weak and the energies of the corresponding discrete quantum states for neutrons are so low (of the scale of $1 \mathrm{peV}$ ) that the observation of such states is indeed a major experimental challenge. What is more, the measurements in this energy range open the door, for instance, to experimental studies of the fundamental short-range forces for neutrons near solid surfaces (see [3,4] and references therein). This unique opportunity is inherent to the high sensitivity of the neutron wave functions in the gravitationally bound quantum states of neutrons to the existence of any new type of short-range forces between a neutron and a surface.

The schematics of a typical experiment is the following. A collimated beam of ultracold neutrons is sent between two, usually horizontal, solid plates at a distance several micrometers from each other. The material of the plates is such that the neutrons hitting the plates with vertical velocity above, approximately, $4 \mathrm{~m} / \mathrm{s}$ get absorbed by the plates, while the particles with a lower vertical component of velocity get reflected. One of the plates, usually the upper one, has a rough surface, while the other one is an almost ideal mirror. The quality of the mirror is surprisingly good: the singlereflection losses are in the range $10^{-4}-10^{-5}$. As a result of scattering by a rough wall, the direction of the neutron ve-
PACS number(s): 03.75.Be, 03.75.Dg, 03.65.Ta

locity changes and the neutrons can acquire a sufficiently large vertical component of velocity to get absorbed by the walls. Only the particles that have extremely small vertical velocity (low gravitational energy) do not reach the rough scatterer (the upper plate) and, therefore, are not scattered and absorbed. These particles get through the system and are counted by the exit counter. (In practice, the bottom mirror is often made longer than the scatterer or absorber and the entrance to the neutron counter is collimated with a long horizontal narrow slit. With such a configuration, the absorption is not the only source of neutron loss. The purpose of this slit is to avoid counting of neutrons which exit at a large angle to the mirror surface and could otherwise get counted, albeit with a small probability, either directly or after scattering by the spectrometer walls.)

A quantum description of the experiment differs from the classical one above in several important aspects. The motion of neutrons in the gravitational field between the plates is quantized. The lower the energy, the smaller the (vertical) size of the state. The particles in the lowest gravitational states do not reach the scatterer (or, more precisely, reach it with an exponentially low probability), do not scatter, and, therefore, do not get absorbed. With decreasing distance between the plates, the lower and lower states will reach the scatterer and get absorbed. As a result, the neutron count in the case of spatial quantization of neutrons, should, in ideal circumstances, be a stepwise function of the clearance between the plates. The positions of the steps should then provide the calibration for ultralow-energy measurements. In experiment, unfortunately, such a clear-cut stepwise picture does not emerge: though the curves, without doubt, demonstrate the quantization of the gravitational states, the higher gravitational states have, so far, eluded identification. 
One of the main purposes of this paper is to develop a general theory that would cover the experiment, explain the difficulties associated with identification of the higher states, and suggest ways to optimize the experimental regime. Many aspects of the problem have been discussed very recently in Ref. [5] which analyzed potential mechanisms of neutron losses in experiment [1], including the surface roughness as the principal mechanism of nonspecularity of reflection which is ultimately responsible for the neutron depletion. This paper demonstrated that the losses of neutrons from the lowest gravitational states are determined by the neutron tunneling through the gravitational barrier between the classical turning point and the position of the scatterer or absorber.

However, the theory [5] did not provide an explicit quantitative link between the rate of neutron loss and the parameters of the roughness of the neutron waveguide. Since the nonspecularity of reflection by the rough surfaces is an obvious principal source of the neutron depletion in experiment [1], any accurate quantitative description of the phenomenon should express the neutron count directly via the parameters of the random surface roughness. Our results below not only provide such an explicit link, but also suggest what kind of roughness is the most advantageous for experiment.

In order to extract quantitative information from the experiment and, eventually, to study the short-range forces, one needs an accurate theoretical description of quantum diffusion of neutrons along rough walls. Recently we developed a theory of quantum transport in quasi-two-dimensional (quasi-2D) systems with random rough boundaries (see Refs. $[6,7]$ and references therein). In this paper we apply this formalism to quantized neutrons in the gravitational field. The practical purpose is twofold: to develop an accurate quantitative theory of neutron transport in the presence of random surfaces, which can be used for a quantitative analysis of experimental data, and to determine the parameters of surface roughness for the scatterer surface that would help to optimize future experiments. As for the purely theoretical benefits, we are able to extend our general theory to include strong, high-aperture roughness and to develop a theoretical description for systems with absorbing, "leaky" walls.

Roughness of the walls affects the quantized, in this case, gravitational, states of particles inside the quantum wells by shifting and broadening the energy levels. The stronger the roughness, the larger are this shifting and broadening. In this paper we effectively calculate the line broadening which is determined by the roughness-driven transition between the states. Our perturbative approach works as long as the resulting uncertainty in the level positions is smaller than the distance between the levels. The same condition is necessary for the quantitative interpretation of experiments: though strong roughness can make the effect of the gravitational quantization of states more pronounced, the uncertainty in the level positions can render the results unusable for the calibration of the short-range forces.

The strength of roughness is characterized by the ratio of its amplitude $\ell$ to the size of the well $L$ and by the aperture of roughness $\ell / R$, which is determined by the ratio of the amplitude of roughness $\ell$ to its typical lateral scale $R$ (the correlation radius of surface inhomogeneities). Usually, both the line shifts and broadening are small as long as $\ell / L, \ell / R \ll 1$ (see, for example, Ref. [6] and references therein).

In this particular case there could be two exceptions. First, the highest energy levels, which are close to the absorption threshold, could become relatively broad even for a moderate roughness. This is not very important for the results below since in this paper we deal mostly with direct roughnessdriven transitions from the gravitational states into the continuous spectrum above the threshold and can disregard the transitions via the intermediate states. Second, and more important, the effect of surface roughness on the lowest gravitational states is anomalously small because for such states the particle wave functions on the rough walls are exponentially small in the distance between the classical turning point and the wall. Therefore, as long as we can disregard the absorption processes that involve the intermediate and higher levels, the linewidths of the lowest gravitational states remain small and the calculation of the absorption rate can even be extended to stronger, high-aperture roughness. We plan to address neutron absorption that involves the transitions via the intermediate states later.

When interpreting the experimental results, the main uncertainty is introduced by the lack of accurate data on parameters of surface roughness, especially on the type of correlations and the correlation length of surface inhomogeneities. Simple measurements of the average amplitude and the lateral size of roughness are insufficient when one needs to know the shape of the correlation function of surface inhomogeneities which can be extracted, almost exclusively, from diffraction measurements [8]. Another major source of uncertainty is relatively poor information on the energy or velocity distribution of neutrons in the beam. While the dispersion in the horizontal component of velocity can be measured very accurately, the precise measurements of the vertical dispersion are much more problematic. Therefore, one of the goals of this paper is to identify the range of parameters in which this lack of precise input data affects the results as little as possible. Surprisingly, this is possible.

The paper has the following structure. In the next section we describe the neutron wave functions and eigenstates, introduce proper dimensionless variables, and present a set of transport equations for quantized neutrons near random rough wall(s). In Sec. III we present the roughness-driven scattering probabilities in the case of weak roughness. In Sec. IV we explore the ways to generalize our theory to stronger roughness with some success in the case of highaperture low-amplitude roughness. The final results for absorption times for neutrons on the leaky walls and for the neutron count for direct and inverse geometries are given in Sec. V. Sec. VI contains conclusions and discussion of how to improve our theory and optimize the experiment.

\section{MAIN EQUATIONS}

\section{A. Potential well, notations, and dimensionless variables}

In the bulk of the paper we will disregard the ultrashortrange potential for neutrons near solid surfaces and consider surfaces of solid plates as potential barriers for neutrons of the height $U_{c}$ (the absorption barrier). We will discuss 


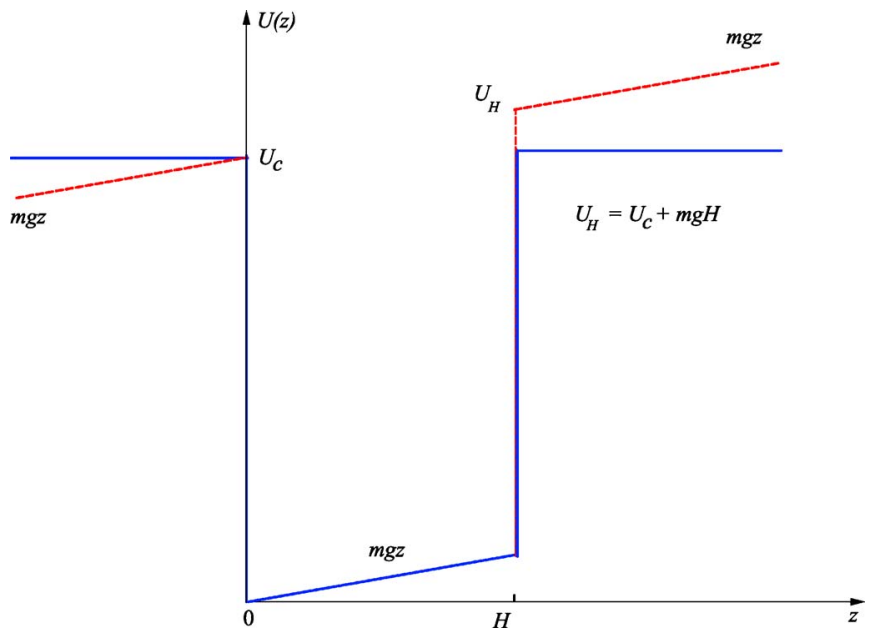

FIG. 1. (Color online) Schematic drawing of the potential well in vertical direction $z$. Dashed lines, the "real" potential; solid lines, the potential used in the paper. Since $m g H / U_{c} \sim 10^{-5}$ and we are particularly interested in the lowest energy levels, the corrections are negligible (with an accurately scaled potential, the difference between the dashed and solid levels cannot be seen).

the short-range forces near the walls toward the end of the paper. If one forgets for a moment about the roughness of the scatterer or absorber (the upper plate), the potential energy for neutrons in the vertical direction, $U(z)$, is $U(z \leq 0)=U_{c}+m g z$ (mirror at the bottom), $U(z)=m g z$ at $0<z<H$ (the gravitational field between the plates; the scatterer/absorber is at the distance $H$ from the bottom mirror), and $U(z)=m g z+U_{c} \equiv U_{H}+m g(z-H)$ (the absorption barrier) at $z \geq H$. Schematically, this potential is presented in Fig. 1 by the dashed lines. An alternative geometry is discussed in Sec. V E.

Numerically, $U_{c} \sim 1.34 \times 10^{-26} \mathrm{~J}$ (the kinetic barrier for absorption is $4 \mathrm{~m} / \mathrm{s}$ ). The characteristic gravitational energies inside the well, on the other hand, are by five orders of magnitude lower, $m g H \sim 1.6 \times 10^{-31} \mathrm{~J}$ (for $H \sim 10 \mu \mathrm{m}$ ). This means that the plot in Fig. 1 is out of scale. Since we are interested almost exclusively in the gravitational states near the bottom of the well, we can, without any noticeable loss of accuracy, ignore the gravitational field near the top of the well and replace the exact potential (the dashed lines) by the one given in Fig. 1 by solid lines.

The energy spectrum of neutrons in this potential well consists of discrete energy states $\epsilon_{n}$ below $U_{c}$ and continuum above. (Note that the short-lived discrete states near the threshold could be also treated as continuum. When the well walls are not ideal but rough, all the energy levels, especially the higher ones, broaden. With an increase in roughness amplitude, lower and lower levels will acquire a noticeable width. This is, in some sense, equivalent to the lowering of the absorption threshold with increasing roughness.) We will call the discrete states close to the bottom of the well the "gravitational states" and higher states in the main part of the well the "square-well states." Though this terminological shortcut is not well defined, it is convenient for discussing the physics and the results because of a very large ratio $U_{c} / m g H \sim 10^{5}$; in computations, unless stated otherwise, all the states will, of course, be treated accurately.

It is convenient to measure the distances $z$ and the energies $\epsilon_{n}$ in units of $l_{0}$ and $e_{0}, \lambda_{n}=\epsilon_{n} / e_{0}$, and $s=z / l_{0}$, where $l_{0}=\hbar^{2 / 3}\left(2 m^{2} g\right)^{-1 / 3} \sim 5.871 \mu \mathrm{m}$ and $e_{0}=m g l_{0} \sim 0.602 \mathrm{peV}$ $\sim 9.6366 \times 10^{-32} \mathrm{~J}$ are the size and the gravitational energy of a neutron in the lowest quantum state in the infinite gravitational trap. In these units, the overall kinetic energy of particles in the beam $E$ and the barrier height $U_{c}$ are $1.4 \times 10^{5}<e=E / e_{0}<8.7 \times 10^{5}, \quad u_{c}=U_{c} / e_{0} \sim 1.4 \times 10^{5} . \mathrm{A}$ very important parameter is the ratio $\chi=u_{c} / e$. In experiment,

$$
0.15 \lesssim \chi \equiv u_{c} / e<1 \text {. }
$$

The value of this parameter shows to what extent the kinetic energy of a neutron exceeds the barrier and determines how easy is it for a neutron to get absorbed by a plate when the direction of velocity is rotated as a result of scattering by the rough scatterer or absorber. As we will see, the results are very sensitive to the exact value of $\chi$ even within the limited range (1) because both values of $u_{c}$ and $e$ are very large.

It is also convenient to introduce the dimensionless velocities (momenta) in the beam direction along the well ( $x$ direction) $\beta_{j}, \quad v_{j}=\beta_{j} v_{0}, \quad v_{0}=\sqrt{2 g l_{0}} \sim 1.073 \times 10^{-2} \mathrm{~m} / \mathrm{s}$, $\beta_{j}=\sqrt{e-\lambda_{j}} \equiv p_{j} l_{0} \approx \sqrt{e}\left(1-\frac{1}{2} \lambda_{j} / e\right)=\sqrt{e-\lambda_{j}}$. for lower levels, $\lambda_{j} \ll e$ and $\left.\beta_{j} \simeq \sqrt{e}\left(1-\frac{1}{2} \lambda_{j} / e\right)\right]$. The range of kinetic energies in experiment is $373<\sqrt{e}<932$.

The roughness of the surface of the scatterer or absorber (the upper plate) is characterized by the correlation function of surface inhomogeneities which is determined by the average lateral size (correlation radius) $R$ and the average amplitude (height) $\ell$ of the inhomogeneities (for precise definitions see Sec. III). In dimensionless variables, $r=R / l_{0}$, $\eta=\ell / l_{0}$. It is reasonable to expect that $\eta \lesssim 0.1$ (so far, in experiments [1] $0.03 \leqslant \eta \leqslant 0.1$; roughness with higher amplitude than 0.1 would be too strong for a quantitative theory). We will discuss how to introduce the surface roughness into the equations later on.

\section{B. Wave functions and energies of the bound states}

At $E<U_{c}$ (or $\left.\lambda_{n}<u_{c} \equiv U_{c} / e_{0}\right)$ the wave function $\psi_{n}(s)$, $s=z / l_{0}$, inside the well, $0 \leq s \leq h=H / l_{0}$, is

$$
\psi_{n}(s)=A_{n}\left[\operatorname{Ai}\left(s-\lambda_{n}\right)-S_{n} \operatorname{Bi}\left(s-\lambda_{n}\right)\right] .
$$

Outside the well,

$$
\psi_{n}(s)=\left\{\begin{array}{l}
B_{n} \exp \left[-k_{n}(s-h)\right], \quad s>h, \\
C_{n} \exp \left[k_{n} s\right], \quad s<0, \quad k_{n}=\sqrt{u_{c}-\lambda_{n}} .
\end{array}\right.
$$

The values of parameters $S_{n}$ and the energy eigenvalues $\lambda_{n}$ are determined by the continuity conditions at $s=0$ and $s=h$ :

$$
\begin{gathered}
C_{n}=A_{n}\left[\mathrm{Ai}\left(-\lambda_{n}\right)-S_{n} \mathrm{Bi}\left(-\lambda_{n}\right)\right], \\
B_{n}=A_{n}\left[\operatorname{Ai}\left(h-\lambda_{n}\right)-S_{n} \operatorname{Bi}\left(h-\lambda_{n}\right)\right], \\
C_{n} k_{n}=A_{n}\left[\mathrm{Ai}^{\prime}\left(-\lambda_{n}\right)-S_{n} \mathrm{Bi}^{\prime}\left(-\lambda_{n}\right)\right], \\
B_{n} k_{n}=-A_{n}\left[\mathrm{Ai}^{\prime}\left(h-\lambda_{n}\right)-S_{n} \mathrm{Bi}^{\prime}\left(h-\lambda_{n}\right)\right],
\end{gathered}
$$

which are equivalent to two equations 


$$
\begin{aligned}
1 / k_{n}= & {\left[\mathrm{Ai}\left(-\lambda_{n}\right)-S_{n} \mathrm{Bi}\left(-\lambda_{n}\right)\right] /\left[\mathrm{Ai}^{\prime}\left(-\lambda_{n}\right)-S_{n} \mathrm{Bi}^{\prime}\left(-\lambda_{n}\right)\right] } \\
= & -\left[\operatorname{Ai}\left(h-\lambda_{n}\right)-S_{n} \mathrm{Bi}\left(h-\lambda_{n}\right)\right] /\left[\mathrm{Ai}^{\prime}\left(h-\lambda_{n}\right)\right. \\
& \left.-S_{n} \mathrm{Bi}^{\prime}\left(h-\lambda_{n}\right)\right] .
\end{aligned}
$$

Computation of the neutron count would also require the normalization coefficients $A_{n}$,

$$
\begin{aligned}
A_{n}^{-2}= & l_{0} \int_{0}^{h}\left[\operatorname{Ai}\left(s-\lambda_{n}\right)-S_{n} \operatorname{Bi}\left(s-\lambda_{n}\right)\right]^{2} d s+\frac{l_{0}}{2 k_{n}}\left\{\left[\operatorname{Ai}\left(-\lambda_{n}\right)\right.\right. \\
& \left.\left.-S_{n} \operatorname{Bi}\left(-\lambda_{n}\right)\right]^{2}+\left[\operatorname{Ai}\left(h-\lambda_{n}\right)-S_{n} \operatorname{Bi}\left(h-\lambda_{n}\right)\right]^{2}\right\}
\end{aligned}
$$

and $B_{n}, C_{n}$, Eq. (4). Some information on discrete states can also be found in Ref. [5].

\section{Approximate description of the bound states}

For what follows, we will not always need the exact eigenvalues and the wave functions. First, let us note that the threshold energy $U_{c}$ is much higher (by about five orders of magnitude) than the gravitational energy $m g H$ in a typical experiment, $u_{c} \gg h$. One can also separate the states into two groups: the lowest states for which the presence of the gravitational field near the bottom of the well is important (the gravitational states) and the higher states for which the fact that the gravity determines the shape of the bottom of the well is mostly irrelevant (the square-well states).

For the gravitational states in an infinite well, $u_{c} \rightarrow \infty$, the wave functions (2) should become equal to zero on the walls $s=0 ; h$, and equations for the eigenvalues Eq. (5) should be replaced by the following equation for the energy spectrum $\bar{\lambda}_{n}(h)$ (here and below we identify physical parameters, which are calculated for a well with infinite walls, by a bar over the corresponding symbols):

$$
\bar{S}_{n}=\operatorname{Ai}\left(-\bar{\lambda}_{n}\right) / \operatorname{Bi}\left(-\bar{\lambda}_{n}\right), \quad \operatorname{Ai}\left(h-\bar{\lambda}_{n}\right)-\bar{S}_{n} \operatorname{Bi}\left(h-\bar{\lambda}_{n}\right)=0 .
$$

The normalizing coefficients $A_{n}$ are now defined as [9]

$$
\begin{gathered}
\bar{A}_{n}^{2}=\frac{1}{l_{0}} a_{n}, \\
a_{n}=\left(\int_{0}^{h}\left[\mathrm{Ai}\left(s-\bar{\lambda}_{n}\right)-\bar{S}_{n} \mathrm{Bi}\left(s-\bar{\lambda}_{n}\right)\right]^{2} d s\right)^{-1} \\
=\left\{\left[\mathrm{Ai}^{\prime}\left(-\bar{\lambda}_{n}\right)-\bar{S}_{n} \mathrm{Bi}^{\prime}\left(-\bar{\lambda}_{n}\right)\right]^{2}\right. \\
\left.-\left[\mathrm{Ai}^{\prime}\left(h-\bar{\lambda}_{n}\right)-\bar{S}_{n} \mathrm{Bi}^{\prime}\left(h-\bar{\lambda}_{n}\right)\right]^{2}\right\}^{-1} .
\end{gathered}
$$

If for the lowest gravitational states the size of the state $\lambda_{n}$ is much smaller than the spacing between the walls, $\lambda_{n} \ll h$, the equation for the energy spectrum is even simpler,

$$
\operatorname{Ai}\left(-\tilde{\lambda}_{n}\right)=0
$$

with the semiclassical eigenvalues

$$
\tilde{\lambda}_{n}^{s c}=\left[\frac{3 \pi}{4}\left(2 n-\frac{1}{2}\right)\right]^{2 / 3}
$$

and the normalizing coefficients

$$
\tilde{A}_{n}^{2}=\frac{1}{l_{0}} \tilde{a}_{n}, \quad \tilde{a}_{n}=\left(\int_{0}^{\infty} \operatorname{Ai}\left(s-\tilde{\lambda}_{n}\right)^{2} d s\right)^{-1}=\frac{1}{l_{0}} \operatorname{Ai}^{\prime-2}\left(-\tilde{\lambda}_{n}\right) .
$$

Since $u_{c}$ is very large but finite, $u_{c} \sim 10^{5}$, the eigenvalues $\lambda$ differ from their values $\bar{\lambda}$ [Eq. (7)] at $u_{c} \rightarrow \infty, \lambda=\bar{\lambda}+\delta \lambda$, $S=\bar{S}+\delta S$. The corresponding corrections can be calculated by expanding Eqs. (5) in $1 / \sqrt{u_{c}-\bar{\lambda}}$ :

$$
\begin{aligned}
& -\frac{1}{\sqrt{u_{c}-\bar{\lambda}}}=\delta \lambda+\delta S \frac{\mathrm{Bi}(-\bar{\lambda})}{\mathrm{Ai}^{\prime}(-\bar{\lambda})-\bar{S} \mathrm{Bi}^{\prime}(-\bar{\lambda})}, \\
& \frac{1}{\sqrt{u_{c}-\bar{\lambda}}}=\delta \lambda+\delta S \frac{\mathrm{Bi}(h-\bar{\lambda})}{\mathrm{Ai}^{\prime}(h-\bar{\lambda})-\bar{S} \mathrm{Bi}^{\prime}(h-\bar{\lambda})} .
\end{aligned}
$$

Then the normalizing coefficients $B, C(\bar{B}=\bar{C}=0)$ become

$$
\begin{gathered}
B=\psi(H)=-\bar{A}\left[\mathrm{Ai}^{\prime}(h-\bar{\lambda})-\bar{S} \mathrm{Bi}^{\prime}(h-\bar{\lambda})\right] / \sqrt{u_{c}-\bar{\lambda}}, \\
C=\psi(0)=\bar{A}\left[\mathrm{Ai}^{\prime}(-\bar{\lambda})-\bar{S} \mathrm{Bi}^{\prime}(-\bar{\lambda})\right] / \sqrt{u_{c}-\bar{\lambda}} .
\end{gathered}
$$

For the square-well states well above the bottom, but still below $U_{c}$, one can neglect the gravitational field near the bottom and use the standard equation for the eigenvalues $\lambda_{n}$,

$$
\begin{gathered}
\tan (\sqrt{\lambda} h)=-\frac{\sqrt{\lambda}+k S}{k-\sqrt{\lambda} S}, \\
k \equiv \sqrt{u_{c}-\lambda}, \quad S \equiv \sqrt{\lambda /\left(u_{c}-\lambda\right)} .
\end{gathered}
$$

For these symmetric states the values of the wave functions on the walls are

$$
\begin{gathered}
\psi_{n}^{2}(0)=\psi_{n}^{2}(H)=A^{2} S^{2}, \\
A^{-2}=\frac{l_{0}}{k} S^{2}+\frac{l_{0}}{4 \sqrt{\lambda}}\left[2(\sqrt{\lambda} h+S+\sqrt{\lambda} h S)^{2}\right. \\
\left.-2 S \cos (2 \sqrt{\lambda} h)-\left(1-S^{2}\right) \sin (2 \sqrt{\lambda} h)\right] .
\end{gathered}
$$

In the case of very deep levels $\lambda / u_{c}$, Eqs. (13)-(15) can be simplified as

$$
\begin{gathered}
\lambda=\bar{\lambda}\left(1-\frac{4}{h \sqrt{u_{c}}}\right), \quad S=\sqrt{\frac{\overline{\bar{\lambda}}}{u_{c}}}, \\
\psi_{n}^{2}(0)=\psi_{n}^{2}(H)=\frac{2}{l_{0} h} \frac{\bar{\lambda}}{u_{c}},
\end{gathered}
$$

where $\bar{\lambda}$ and $\bar{A}$ are the eigenvalues and the normalizing coefficient for the infinitely deep well,

$$
\bar{\lambda}_{n}=\pi^{2} n^{2} / h^{2}, \quad \bar{A}_{n}^{2}=1 /\left(2 l_{0} h\right) .
$$

Approximately, the total number of discrete levels is

$$
\mathcal{N} \sim \frac{h}{\pi} \sqrt{u_{c}} \approx 120 h .
$$




\section{Time evolution of the neutron population}

Recently we developed a versatile formalism for description of 2D diffusion of quantized particles along random rough walls [6]. The key is to include the information on the wall roughness not into the boundary conditions, but into the roughness-driven transition probabilities between the quantum states in the transport equation. The application of this formalism to the neutron problem at hand requires several important modifications: we should include neutron absorption by the "leaky" walls and reconsider the time and space properties of the transport equation.

The possibility of neutron absorption by the walls can be taken into account by the calculation of the probability of transitions into the continuous spectrum without the counterbalancing terms in the transport equation. The second anomaly of this problem is that we are calculating not a stationary diffusion flow but the time dynamics of the neutron count. As a result, we have to solve a time-dependent problem with an initial condition in the form of initial distribution of neutrons over the vertical velocities, i.e., over the gravitational quantum states.

The main modification deals with the dimensionality of the problem. In [6] we considered the scattering-driven $2 \mathrm{D}$ diffusion in two equivalent directions parallel to the walls while the third dimension (the finite motion perpendicular to the plates) gave rise to a set of discrete quantum states. In our neutron problem there is a preferred direction along the plates (the beam direction $x$ ); the neutron count is being done only in this direction. Therefore, we should exclude the sideways motion and reformulate the transport problem as a $2 \mathrm{D}$ problem with vertical motion (coordinate $z$ ) and the direction of the beam ( $x$ direction with the corresponding momentum $p)$. In other words, we have to modify the transport equation of Refs. [6,7] (equations for the particle balance) for quasi-2D particles (particles with continuous spectrum in the $x, y$ directions and discrete quantized gravitational states in the $z$ direction) in order to eliminate the momentum in the $y$ direction, $q$.

According to [6], the distribution functions $n_{j}(q, p)$ in each quantized state $j$ obey the following Boltzmann equations:

$$
\begin{aligned}
\frac{d n_{j}}{d t}= & \frac{2 \pi}{\hbar} \sum_{j^{\prime}} \int W_{j j^{\prime}}\left(q, p ; q^{\prime}, p^{\prime}\right)\left(n_{j^{\prime}}-n_{j}\right) \\
& \times \delta\left(\epsilon_{j q p}-\epsilon_{j^{\prime} q^{\prime} p^{\prime}}\right) \frac{d q^{\prime} d p^{\prime}}{(2 \pi)^{2}}
\end{aligned}
$$

where the $2 \mathrm{D}$ vector $\mathbf{q}$ in the plane of the mirror has $y$ and $x$ components $\mathbf{q}=(q, p)$. Since we disregard the change of momentum in the $y$ direction,

$$
\begin{gathered}
W_{j j^{\prime}}\left(\mathbf{q}, \mathbf{q}^{\prime}\right)=\hbar \delta\left(q-q^{\prime}\right) W_{j j^{\prime}}\left(p, p^{\prime}\right), \\
n_{j}(q, p)=\hbar \frac{2 \pi}{L_{y}} \delta(q) n_{j}(p),
\end{gathered}
$$

and the integration over $d q^{\prime}$ in Eq. (20) yields

$$
\begin{aligned}
\frac{d n_{j}(p)}{d t}= & 2 \pi \sum_{, j^{\prime}} \int W_{j j^{\prime}}\left(p, p^{\prime}\right)\left[n_{j^{\prime}}\left(p^{\prime}\right)-n_{j}(p)\right] \\
& \times \delta\left(p^{\prime 2} / 2 m-p^{2} / 2 m+\epsilon_{j^{\prime}}-\epsilon_{j}\right) \frac{d p^{\prime}}{2 \pi} .
\end{aligned}
$$

We are dealing with particles with a fixed energy $E$, i.e., with particles in gravitational quantum states $\epsilon_{j}$ with distinct values of the lateral momentum $p_{j}=\sqrt{2 m\left(E-\epsilon_{j}\right)}$,

$$
n_{j}(p)=\frac{2 \pi \hbar}{L_{x}} \delta\left(p-p_{j}\right) N_{j},
$$

where $N_{j}$ is the number of particles in state $j$ per unit length of the beam. Finally, the equations for the particle balance (22) reduce to

$$
\frac{d N_{j}}{d t}=\sum_{j^{\prime}} W_{j j^{\prime}}\left(p_{j}, p_{j^{\prime}}\right)\left[N_{j^{\prime}} / v_{j^{\prime}}-N_{j} / v_{j}\right]
$$

To this equation we should add the terms responsible for the neutron absorption (transitions into the continuous spectrum):

$$
\partial N_{j} / \partial t=\sum_{\lambda_{j^{\prime}} \leq u_{c}} W_{j j^{\prime}}\left(N_{j^{\prime}} / v_{j^{\prime}}-N_{j} / v_{j}\right)-N_{j} / v_{j} \int_{\lambda^{\prime}>u_{c}} W_{\lambda^{\prime} j} d \lambda^{\prime} .
$$

Note that the separation of the energy spectrum into discrete lines and continuum, which is exact for an ideal well in Fig. 1, is unambiguous only for weak roughness. In general, roughness leads to shifting and broadening of all the lines. The transition probabilities and, therefore, the broadening are expected to be higher for the higher levels (see the next section). With increasing amplitude of roughness, the higher levels can become broad enough to be considered continual, which, in turn, is equivalent to the lowering of the absorption threshold. In the case of the high-amplitude roughness this effect could even become dominant. However, in the case of high-amplitude roughness the whole energetics of the systems changes so dramatically that it could be impossible to use the experimental results in a meaningful quantitative way.

The calculation of the neutron count can be done either via the time dependence of the neutron number at fixed position during the time of flight $t=L / v_{x}$ or via the decrease of the number of neutrons while they move along the absorber of the length $L=t v_{x}$. Within the former approach, if initially there was $N_{j}(0)$ neutrons per unit area in each discrete state, the time evolution of the densities $N_{j}$ is described by the particle balance equations (25).

The set of linear differential equations (25) describes the exponential disappearance of neutron with a set of characteristic relaxation times $\tau$. These relaxation times $\tau$ are given, according to Eq. (25), by the following characteristic equation: 


$$
\begin{gathered}
0=\operatorname{det}\left|\left(\frac{1}{\tau}-\frac{1}{\tau_{j}^{(0)}}\right) \delta_{j j^{\prime}}+\frac{1}{\tau_{j j^{\prime}}}\right|, \\
\frac{1}{\tau_{j}^{(0)}}=\frac{1}{v_{j}} \int_{\lambda^{\prime}>u_{h}} W_{\lambda^{\prime} j} d \lambda^{\prime}, \quad \frac{1}{\tau_{j j^{\prime}}}=\frac{1}{v_{j^{\prime}}} W_{j j^{\prime}}-\frac{1}{v_{j}} \delta_{j j^{\prime}} \sum W_{j j^{\prime \prime} \leq Z},
\end{gathered}
$$

where it is convenient to introduce the dimensionless velocities $\beta_{j}, v_{j}=\beta_{j} v_{0}, v_{0}=\sqrt{2 g l_{0}} \sim 1.0733 \times 10^{-2} \mathrm{~m} / \mathrm{s}$.

Equation (26) yields $\mathcal{N}$ relaxation times $\tau=\tau_{j}$ and the same number of eigenvectors $\mathbf{N}_{j}$ which evolve as

$$
\mathbf{N}_{j}=\mathbf{N}_{j}(0) \exp \left(-t / \tau_{j}\right) .
$$

The number of particles in each discrete (gravitational) state $N_{i}$ decreases as

$$
N_{i}=\sum_{j} N_{i j}(0) \exp \left(-t / \tau_{j}\right),
$$

where $N_{i j}$ is the $i$ th component of the $j$ th eigenvector. In other words, we have to project the initial distribution of neutrons over the gravitational states $\left(N_{1}(0), N_{1}(0), \ldots\right)$ on the eigenvectors $\mathbf{N}_{j}$. Then the overall neutron count is

$$
N(h)=\sum_{i, j} N_{i j}(0) \exp \left[-L_{x} / V_{0} \tau_{j}(h)\right],
$$

where the time is limited by the time of flight between the plates $t=L_{x} / V_{0} \sim 2 \times 10^{-2} \mathrm{~s}$. The dependence $N(h)$ acquires a distinct structure only if there is a pronounced hierarchy in the set of relaxation times $\tau_{j}(h)$ and if there are pronounced changes in the longest relaxation times $\tau_{j}(h)$ as a function of the separation between the plates.

It is clear that we should take into account only the relaxation times that are comparable to or much bigger than the time of flight $t=L_{x} / V_{0}$. For much shorter relaxation times we do not have to do any precise calculations and just disregard the components of the initial distribution which are decaying as the corresponding eigenvectors.

As we will see below, these longest relaxation times $\tau_{j}(h)$, which correspond to the particles in the lowest-energy states far away from the absorption edge, experience rather steep changes at certain values of the spacings between the plates $h_{1}<h_{2}<h_{3} \cdots$. However, this feature can be observed only for specific types of the roughness of the scatterer surface when the values $h_{1}, h_{2}, h_{3}, \ldots$ are sufficiently far away from each other. One of the crucial elements here is the relation between the times $\tau_{j j^{\prime}}$ and $\tau_{j}^{(0)}$. If $\tau_{j j^{\prime}}$ is shorter than $\tau_{j}^{(0)}$, then the loss of neutrons occurs as a result of scattering-driven gradual increase in gravitational energy (slow diffusion of particles between the states $j$ with a gradual increase in the state index $j$ ) until the neutron gets into the absorber. If this is the case, the disappearance times for neutrons from the neighboring states are comparable and it is not easy to extract the information on the properties of quantized gravitational states from the neutron count.

In the opposite case of very short times $\tau_{j}^{(0)}$, the neutron gets absorbed essentially as a result of a single scattering act. In this case, one can disregard the interstate transitions and $\tau_{j} \simeq \tau_{j}^{(0)}$. This regime is more advantageous for experiment especially if there is a distinct hierarchy of times $\tau_{j}^{(0)}$. The transition between these regimes is determined by the lateral size (correlation radius) of surface inhomogeneities and the asymptotic behavior of the correlation function. All this information is contained in the transition probabilities $W_{j j^{\prime}}\left(p_{j}, p_{j^{\prime}}\right)$. In what follows we will pay particular attention to the experimental conditions necessary for the observation of the proper regime.

Note that the transitions between the states are largely suppressed, $\tau_{j \neq j^{\prime}} \gg \tau_{j j}$, when the lateral size of the surface inhomogeneities on the scatterer $R$ is much larger than the size of quantized states (in this case, $R \gg l_{0}$, or, in dimensionless units, $r \equiv R / l_{0} \gg 1$ ) [6]. Since the momentum change $\delta \beta$ as a result of scattering by inhomogeneities of the size $r$ is of the order of $\delta \beta \sim 1 / r$ and is very small for large $r$, it seems that in this case the interstate transitions are allowed only when the energy gaps between the states are small. If this were correct, the population of the higher states would have remained constant no matter how long one waited (the gaps between the states increase with increasing state number $j$ approximately proportionally to $j$ ). However, this is not completely true because even at $r \equiv R / l_{0} \gg 1$ there are certain values of the spacings between the plates $h_{j}$ at which the transition channels $j \leftrightarrow j+1$ open spontaneously [7] leading to a drastic decrease in the relaxation times: the scatteringdriven momentum change $\delta \beta \sim 1 / r$ is sufficient for the transition between the states $j$ and $j^{\prime}$ when

$$
\beta_{j}-\beta_{j+1} \simeq \frac{1}{2}\left(\lambda_{j+1}-\lambda_{j}\right) / \sqrt{e} \simeq 1 / r
$$

or, in normal variables, when the spacing $H$ satisfies the equation

$$
\frac{1}{2}\left[\epsilon_{j+1}(H)-\epsilon_{j}(H)\right] / \sqrt{E e_{0}(H)} \simeq l_{0}(H) / R .
$$

However, it is highly unlikely that this spontaneous opening of mode-coupling channels affects the neutron count in the existing experimental setups. The observation of this effect requires drastically different experiments [10]. To avoid dealing with this issue, we will assume that the size of inhomogeneities $r$ is relatively small.

In order to find the dynamics of the neutron count, we need information about the initial distribution of neutrons over the vertical velocities [over discrete energy states $\left.N_{j}(t=0)\right]$. This distribution reflects the neutron distribution over the vertical velocities in front of the slit and is determined by the expansion of the classical wave functions in front of the slit over the gravitational states. This can, in principle, be done precisely, but only if one knows the exact distribution of particles over the vertical velocities in the initial beam. Unfortunately, at present the distribution of neutrons over the vertical velocities cannot be measured as accurately as the horizontal distribution. However, even if this distribution were available, the exact mathematical problem would still have been too complicated because of the complex structure of the experimental apparatus. 
The analysis of experiment, which has been performed in Ref. [4], indicates that the initial distribution is approximately uniform,

$$
N_{j}(0)=N(0) / Z,
$$

where $N(0)$ is the overall combined density of neutrons in all gravitational states and $Z$ is the total number of such discrete states between the plates with $\epsilon_{j}<U_{C}$. Note that the uniform distribution (32) is a nonequilibrium one and should evolve in time as a result of scattering by surface inhomogeneities even in the absence of the neutron absorption. The equilibrium distribution, which does not evolve as a result of nonabsorbing scattering irrespective of the form of collision operator, is given by equation

$$
N_{j}(0)=N(0) \beta_{j} /\left(Z \sum_{i \leq Z} \beta_{i}\right), \quad N_{j}(0) / \beta_{j}=\text { const. }
$$

Luckily for us, the exit neutron count depends mostly only on the initial population of the lowest states. The lifetime of the neutrons from the higher states is much shorter and they get absorbed well before getting to the counter. The velocities $\beta_{i}$ for the lowest states can be considered as constants, $\beta_{i} \approx \beta_{0}$, and, if there is no singularity in the velocity distribution in the beam at zero angle, the distribution for the lowest states is close to uniform, Eq. (32), anyway. In this case, the difference between the distributions (33) and (32) is negligible as well.

The last remaining issue before solving the transport equations (25) is to relate the transition probabilities $W_{j j^{\prime}}$ to the surface roughness (to the correlation function of surface inhomogeneities).

\section{SCATTERING PROBABILITIES: WEAK ROUGHNESS}

Roughness of (one of) the walls leads to scattering of neutrons, which, in turn, results in the transitions between the states, broadening of the lines, etc. In some intermediate energy range, when the lifetimes are already short but the spacings between levels are not yet large, this broadening can even effectively transform the discrete energy levels into a continuum. However, when the matrix elements are still relatively small, one can always separate the effects of scattering on the energy spectrum from the transition probabilities that affect the particle transport [6] and, in our case, the neutron count.

We will start from the case of slight roughness for which there are well established methods of calculation of the surface-driven scattering probabilities [6] (see also [11,12] and our earlier application to neutrons in a gravitational trap [13]). Since the geometry of the problem is somewhat different from our previous applications [6,13], the calculation should be modified though the general method remains the same.

The rough scatterer corresponds to the barrier of the height $U_{c}$ with position $z=H+\xi(x, y)$, where $\xi(x, y)$ is the random function which describes the surface roughness, instead of its "ideal" position at $z=H$ in Fig. 1. If we neglect the small gravitational potential $m g H$ in comparison with the barrier $U_{c}$ (the difference in scales is $10^{5}$ ), the roughnessdriven "perturbation" becomes

$$
\begin{aligned}
V(z, x, y) & =U_{c} \theta(z-H-\xi)-U_{c} \theta(z-H) \\
& \approx-\xi(x, y) \delta(z-H) U_{c} .
\end{aligned}
$$

The transition probabilities are given by the squares of the matrix elements of this perturbation averaged over the surface inhomogeneities,

$$
W_{j j^{\prime}}\left(\mathbf{q}, \mathbf{q}^{\prime}\right)=\frac{1}{\hbar^{2}}\left\langle\left|V_{j p q, j^{\prime} p^{\prime} q^{\prime}}\right|^{2}\right\rangle_{\xi} .
$$

The matrix elements should be calculated using the wave functions

$$
\Psi=\psi(x, y) \psi_{j}(z)
$$

where $\psi(x, y)$ are the properly normalized plane waves and the functions $\psi_{j}(z)$ are given by Eqs. (2)-(6). [6])

The matrix elements of the perturbation (34) are (cf. Ref.

$$
\begin{aligned}
V_{j j^{\prime}} \equiv & \int \exp \left[i x\left(p-p^{\prime}\right) / \hbar+i y\left(q-q^{\prime}\right) / \hbar\right] \xi(x) \psi_{j}(z) \psi_{j^{\prime}}(z) \\
& \times\left(m g H-U_{c}\right) \delta(z-H) d x d s \\
= & -\xi\left(p-p^{\prime}, q-q^{\prime}\right) U_{c} \psi_{j}(H) \psi_{j^{\prime}}(H) .
\end{aligned}
$$

The scattering probabilities (35) are then

$$
\begin{aligned}
W_{j j^{\prime}}\left(p, q ; p^{\prime}, q^{\prime}\right) & =\frac{1}{\hbar^{2}}\left\langle\left|V_{j p q, j^{\prime} p^{\prime} q^{\prime}}\right|^{2}\right\rangle_{\xi} \\
& =\frac{1}{\hbar^{2}} \zeta\left(p-p^{\prime}, q-q^{\prime}\right) U_{c}^{2} \psi_{j}^{2}(H) \psi_{j^{\prime}}^{2}(H)
\end{aligned}
$$

where $\zeta\left(p-p^{\prime}, q-q^{\prime}\right)$-the so-called power spectrum of inhomogeneities-is the Fourier image of the correlation function of surface inhomogeneities $\zeta(x, y)$,

$$
\begin{aligned}
\zeta(x, y) & \equiv\left\langle\xi\left(x_{1}, y_{1}\right) \xi\left(x_{1}+x, y_{1}+y\right)\right\rangle \\
& \equiv A^{-1} \int \xi\left(x_{1}, y_{1}\right) \xi\left(x_{1}+x, y_{1}+y\right) d x_{1} d y_{1},
\end{aligned}
$$

and $A$ is the averaging area. Finally, assuming the homogeneity of the surface in the $y$ direction, i.e., the lack of the dependence of $\zeta(x, y)$ on $y$, we get

$$
\begin{gathered}
W_{j j^{\prime}}\left(p, q ; p^{\prime}, q^{\prime}\right)=\delta\left(q-q^{\prime}\right) W_{j j^{\prime}}\left(p, p^{\prime}\right), \\
W_{j j^{\prime}}\left(p, p^{\prime}\right)=\frac{1}{\hbar^{2}} \zeta\left(p-p^{\prime}\right) U_{c}^{2} \psi_{j}^{2}(H) \psi_{j^{\prime}}^{2}(H) .
\end{gathered}
$$

For computations, we will use the most common Gaussian correlation function,

$$
\zeta(x)=\ell^{2} \exp \left(-x^{2} / 2 R^{2}\right), \quad \zeta(p)=\sqrt{2 \pi} \ell^{2} R \exp \left(-p^{2} R^{2} / 2\right),
$$

where the amplitude $\ell$ and the correlation radius $R$ characterize the averaged height and lateral size of surface inhomo- 
geneities. Note that though in many cases the real rough surface can be different from the Gaussian one $[8,14]$, there are reasons to believe that the roughness in experiment [1] is close to Gaussian. The results for the relaxation time can also be sensitive to the type of the asymptotic decay of the power spectrum of inhomogeneities at large $p$ [7]. Below we will choose such a regime for which the interpretation of experimental data would be the least sensitive to this uncertainty in parameters of the surface correlator.

In our dimensionless variables, the transition probability (39), (40) acquires the form

$$
W_{j j^{\prime}}=\frac{\sqrt{2 \pi}}{\hbar^{2}} e_{0}^{2} l_{0}^{3} \eta^{2} r \exp \left[-\left(\beta_{j}-\beta_{j^{\prime}}\right)^{2} r^{2} / 2\right] u_{c}^{2} \psi_{j}^{2}(h) \psi_{j^{\prime}}^{2}(h),
$$

where $\eta=\ell / l_{0}$ and $r=R / l_{0}$. The condition of weak roughness is

$$
\eta \equiv \ell / l_{0} \ll 1, r .
$$

In experiment, the potential barrier $U_{c}$ is very high and can be considered infinite in calculations of the roughnessdriven scattering probabilities for transitions between the lower gravitational states. In this case, Eq. (36) should be replaced by

$$
V_{j j^{\prime}}=-\frac{\hbar^{2}}{2 m} \xi\left(p-p^{\prime}, q-q^{\prime}\right) \psi_{j}^{\prime}(H) \psi_{j^{\prime}}^{\prime}(H)
$$

and Eq. (41) by

$$
\begin{aligned}
& W_{j j^{\prime}}\left(p_{j}-p_{j^{\prime}}\right) \\
& \quad=\frac{\sqrt{2 \pi} \hbar^{2}}{4 m^{2}} l_{0}^{3} \eta^{2} r \exp \left[-\left(\beta_{j}-\beta_{j^{\prime}}\right)^{2} r^{2} / 2\right] \psi_{j}^{\prime 2}(H) \psi_{j^{\prime}}^{\prime 2}{ }^{2}(H) \\
& \quad=\frac{1}{\tau_{0}} w_{j j^{\prime}},
\end{aligned}
$$

where we introduce the following scale $\tau_{0}$ for transition times:

$$
\frac{1}{\tau_{0}}=\frac{\sqrt{2 \pi}}{4 m^{2}} \frac{\hbar^{2}}{l_{0}^{3} v_{0}} \eta^{2}=\frac{\sqrt{2 \pi}}{4 m} \frac{\hbar}{l_{0}} \eta^{2}
$$

The form (41) with $U_{c}$ should be used only when calculating the transitions that involve the upper energy levels and the continuous spectrum above the threshold.

For the gravitational states (7), the transitional probabilities (44) become

$$
\begin{aligned}
W_{j j^{\prime}}\left(p_{j}-p_{j^{\prime}}\right)= & \frac{1}{\tau_{0}} w_{j j^{\prime}}=\frac{\sqrt{2 \pi} \hbar^{2}}{4 m^{2}} \frac{\eta^{2} r}{l_{0}^{3}} \exp \left[-\left(\beta_{j}-\beta_{j^{\prime}}\right)^{2} r^{2} / 2\right] \\
& \times a_{j}^{2} a_{j^{\prime}}^{2}\left[\mathrm{Ai}^{\prime}\left(h-\bar{\lambda}_{j}\right)-S \mathrm{Bi}^{\prime}\left(h-\bar{\lambda}_{j}\right)\right]^{2} \\
& \times\left[\mathrm{Ai}^{\prime}\left(h-\bar{\lambda}_{j^{\prime}}\right)-S \mathrm{Bi}^{\prime}\left(h-\bar{\lambda}_{j^{\prime}}\right)\right]^{2}
\end{aligned}
$$

with $A_{j}^{2}$ given by Eq. (8).

For the square-well states (18), the scattering probabilities acquire the "standard" form [6]

$$
\begin{aligned}
W_{j j^{\prime}}\left(p_{j}-p_{j^{\prime}}\right) & =\frac{1}{\tau_{0}} w_{j j^{\prime}} \\
& =\frac{\sqrt{2 \pi} \pi^{4} \hbar^{2}}{16 m^{2}} \frac{\eta^{2} r}{l_{0}^{3}} \exp \left[-\left(\beta_{j}-\beta_{j^{\prime}}\right)^{2} r^{2} / 2\right] \frac{j^{2} j^{\prime 2}}{h^{6}}
\end{aligned}
$$

We also need the probability of transitions between the gravitational state $j$ and the square-well state $j^{\prime}$ :

$$
\begin{aligned}
W_{j j^{\prime}}\left(p_{j}-p_{j^{\prime}}\right)= & \frac{1}{\tau_{0}} w_{j j^{\prime}} \\
= & \frac{\sqrt{2 \pi} \pi^{2} \hbar^{2}}{8 m^{2}} \frac{\eta^{2} r}{l_{0}^{3}} \exp \left[-\left(\beta_{j}-\beta_{j^{\prime}}\right)^{2} r^{2} / 2\right] \frac{j^{\prime 2}}{h^{3}} \\
& \times a_{j}^{2}\left[\mathrm{Ai}^{\prime}\left(h-\lambda_{j}\right)-S \mathrm{Bi}^{\prime}\left(h-\lambda_{j}\right)\right]^{2} .
\end{aligned}
$$

All of the transition probabilities above describe transitions between the discrete states. In order to complete our calculation of the matrix of the relaxation times (26), we have to get the expressions for the transitions from the bound states into the continuous states $1 / \tau_{j}^{(0)}$. To avoid extended calculations, we will first assume that instead of the continuous spectrum we are actually dealing with particles in a large box $L \gg H$ with a set of very close levels $k_{n}=\pi n / L$. Then for the transition probability from discrete states $j$ in our well into the "continuous" spectrum inside the box $L$ one should use Eq. (41),

$$
\begin{gathered}
\frac{1}{\tau_{j}^{(0)}}=\frac{1}{\tau_{0} \beta_{j}} u_{c}^{2} l_{0}^{2} r \psi_{j}^{2}(h) \frac{2}{L_{\lambda_{n}<e}} \sum_{\frac{\exp \left[-\left(\sqrt{e-\lambda_{j}}-\sqrt{e-\lambda_{n}}\right)^{2} r^{2} / 2\right]}{\left[1+\cos ^{2}\left(h \sqrt{\lambda_{n}}\right) /\left(1-u_{c} / \lambda_{n}\right)\right]},} \\
\lambda_{n}=u_{c}+\left(\pi n l_{0} / L\right)^{2} .
\end{gathered}
$$

Switching back from summation over $k_{n}=\pi n / L$ to integration over the continuous energy variable $\lambda$, we get

$$
\begin{aligned}
\frac{1}{\tau_{j}^{(0)}}= & \frac{u_{c}^{2} l_{0} r \psi_{j}^{2}(h)}{\pi \tau_{0} \beta_{j}} \int_{0}^{e-u_{c}} \frac{d \lambda}{\sqrt{\lambda-u_{c}}} \\
& \times \frac{\exp \left[-\left(\sqrt{e-\lambda_{j}}-\sqrt{e-u_{c}-\lambda}\right)^{2} r^{2} / 2\right]}{1+\left(1+u_{c} / \lambda\right) \cos ^{2}\left(h \sqrt{\lambda+u_{c}}\right)} .
\end{aligned}
$$

In principle, the above equations for the transition probabilities are sufficient for solving the transport equations from the previous subsection and for calculating the absorption rates for neutrons in the channel.

\section{TOWARD STRONGER ROUGHNESS}

It seems much more natural to design an experiment with scatterers with strong roughness rather than with the slight one: scattering by strong roughness ensures, almost automatically, a significant turn of the neutron velocity which results in direct absorption of a neutron by a plate. Therefore, neutrons from all states, except for the lowest gravitational ones, for which the wave function does not reach the scatterer, can be absorbed by the plates as a result of, essentially, a single scattering act. Then the count of the exiting neutrons 
will give directly, almost without any ambiguity, the number of particles in the lowest states that are not scattered. In this case, the dependence of the neutron count on the width of the gap between the plates should be the sharpest. If one is interested solely in identification of quantum states, one should definitely try to ensure that the surface roughness is strong. However, if one is interested in extracting the quantitative information from the experiment about the energetics of the system, one should seriously consider using slight roughness for which the particle dynamics can be described very accurately as it is shown above.

In other words, if one is interested solely in observing the quantization of neutrons, one should use a scatterer with strong roughness; if the purpose is the use of quantized states for ultralow-energy calibration, one should use only rough systems which are subjects to a quantitative theory. Therefore, it is important to discuss the restrictions imposed by the conditions of slight roughness and the possibility of extending the limits of applicability of our theory in this particular case to stronger roughness. There are two types of strong roughness: high-amplitude and high-aperture roughness. As we will see, our theory can be extended to the high-aperture low-amplitude roughness, but not to the high-amplitude roughness. In the last section we will argue that the highamplitude roughness should not be used in experiment either.

The surface roughness is considered to be strong if one of the conditions of slight roughness,

$$
\eta \equiv \ell / l_{0} \ll 1, r,
$$

breaks down. In such cases, one cannot usually say much except that one surface collision is sufficient for a complete dephasing of a particle. In our problem such an outcome could even have been looked at as beneficial since this would immediately allow us to estimate the corresponding relaxation time approximately as

$$
\tau_{j}=H / v_{z j} .
$$

However, such an assertion is not always correct since the scattering probability $W$ depends not only on the parameters of the surface (the correlation function of surface roughness

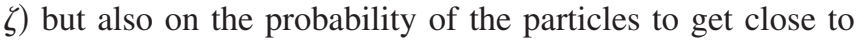
the wall which enters $W$ via the particle wave functions and/or their derivatives on the wall (see, e.g., Eqs. (41) and (44) for $W$ for weak roughness). When such a probability is low, one can (and should) sometimes deal with the strong roughness as if it is weak.

The calculation of the scattering probability consists of two steps: calculation of the matrix elements of the roughness-driven perturbation $V_{j j^{\prime}}$ and the calculation of the scattering probabilities $W$ using these matrix elements. Since the latter step is easier to analyze, we will start from it.

This second step is also less restrictive. Here one has to express the scattering probability $W$ via the matrix elements $V_{j j^{\prime}}$. The scattering probability is always given by the expression of the type (35), but with the scattering $T$ matrix $\hat{T}$ instead of the potential distortion $\widehat{V}$. The link between these two matrices is given by the operator equation

$$
\widehat{T}=\widehat{V}+\widehat{T} \widehat{G} \widehat{V}
$$

where $\widehat{G}$ is the Green's function. For quantized gravitationallike states $j, j^{\prime}$ (the lower states with $h-\lambda_{j, j^{\prime}} \gg 1$ ), the position of the wall is deep under the barrier where the wave functions are attenuating exponentially, roughly as $\exp \left[-(2 / 3)(h-\lambda)^{3 / 2}\right]$. Then the matrix elements of $\widehat{V}$ are exponentially small and Eq. (53) leads to $\widehat{T} \approx \hat{V}$ resulting in the validation of Eq. (35) even when the distortion $V$, by itself, is not small. This means that Eq. (35) can be used as long as we are interested solely in the transitions to and from the gravitational states for which the matrix elements are always small even for not very small roughness. One should be much more careful with the transitions between the higher states.

The simplest way of evaluating the matrix elements $V_{j j^{\prime}}$ seems to be a generalization of approach [11] (see also the first Ref. [6]) for the roughness-driven perturbation

$$
V(z, x, y)=U_{c} \theta(z-H-\xi)-U_{c} \theta(z-H) .
$$

Instead of using Eq. (34), we now write $V$ as an expansion,

$$
V(z, x, y)=U_{c} \sum_{1}^{\infty} \frac{(-1)^{n}}{n !} \delta^{(n-1)}(z-H) \xi^{n}(x, y),
$$

with the matrix elements

$$
\begin{aligned}
V_{j j^{\prime}}\left(\mathbf{q}-\mathbf{q}^{\prime}\right)= & U_{c} \sum_{1}^{\infty} \frac{(-1)^{n}}{n !} \int_{0}^{H} \psi_{j}(z) \delta^{(n-1)}(z-H) \psi_{j^{\prime}}(z) d z \\
& \times \int \xi^{n}(\mathbf{s}) \exp \left[i\left(\mathbf{q}-\mathbf{q}^{\prime}\right) \cdot \mathbf{s}\right] d \mathbf{s} .
\end{aligned}
$$

The first integral reduces to a set of higher derivatives of the wave functions on the wall, $\psi_{j}^{(m)}(H) \psi_{j^{\prime}}^{(n-1-m)}(H)$, or, in dimensionless variables, to $l_{0}^{-n+1} \psi_{j}^{(m)}(h) \psi_{j^{\prime}}^{(n-1-m)}(h)$. Therefore, if the amplitude of the corrugation is $\ell$, the expansion (56) becomes an expansion in $\ell / l_{0}$. If the wave functions on the walls are (exponentially) small, as it is for the lowest gravitational states, the matrix elements $V_{j j^{\prime}}$ can remain small even for not very small values of the corrugation amplitude $\ell$. However, in this case the all the constants in the expressions for the scattering probabilities change: instead of the wave functions on the walls one should write a proper combinations of $\psi_{j}^{(m)}(h)$ from Eq. (56) and, more importantly, the correlation function of the surface corrugation should be replaced by the Fourier images of the higher moments of the correlation.

If, on the other hand, the amplitude of the corrugation is small, $\ell \ll l_{0}$, Eq. (56) leads to exactly the same expression for the matrix elements as Eqs. (36) and, therefore, to the scattering probabilities (35), (41), (44) even when the aperture of the inhomogeneities is large. This means that, as long as the matrices $\widehat{V}$ are small and are close to the $\hat{T}$ matrix, $\widehat{V} \approx \hat{T}$, the high-aperture roughness could still be treated in the same way as weak roughness while the high-amplitude roughness cannot. 
The above analysis on the basis of a generalized approach [11] is not completely accurate. A more accurate analysis can be done using the mapping transformation method (see both Refs. [6]), which involves the lateral derivatives of the surface roughness in a more natural way than the method of Ref. [11]. This method involves the first lateral derivatives $\xi_{x, y}^{(1)}$, which have the order of magnitude $\ell / R$ and not $\ell / l_{0}$, explicitly. As a result, it looks as if the expressions for the matrix elements $V_{j j^{\prime}}$ for the high-aperture roughness are different from Eq. (36). However, the collision operator in the transport equation involves not all the matrix elements of $V_{j j^{\prime}}\left(\mathbf{q}, \mathbf{q}^{\prime}\right)$, but, since the scattering probabilities are always accompanied by the energy $\delta$ functions $\delta\left(E-E^{\prime}\right)$, only those that satisfy the energy conservation law. This means that Eq. (35) can be rewritten for our purposes as

$$
\begin{aligned}
& W_{j j^{\prime}}\left(\mathbf{q}, \mathbf{q}^{\prime}\right) \delta\left(\epsilon_{j}+\frac{q^{2}}{2 m}-\epsilon_{j^{\prime}}-\frac{q^{\prime 2}}{2 m}\right) \\
& =\frac{1}{\hbar^{2}}\left\langle\left|V_{j p q, j^{\prime} p^{\prime} q^{\prime}}\right|^{2}\right\rangle_{\xi} \delta\left(\epsilon_{j}+\frac{q^{2}}{2 m}-\epsilon_{j^{\prime}}-\frac{q^{\prime 2}}{2 m}\right) .
\end{aligned}
$$

It is relatively easy to see [6] that the terms with the derivatives of $\xi$ contribute only to the matrix elements with different energies $E$ and $E^{\prime}$ if the amplitude of roughness is small, $\ell \ll l_{0}$. Therefore, the expressions for the matrix elements at $E=E^{\prime}$ keep their weak-roughness form even for the strong roughness with high aperture, but with low amplitude.

Summarizing, the scattering probabilities remain the same as long as the amplitude of roughness $\eta=\ell / l_{0}$ is small, while the aperture of roughness $\eta / r=\ell / R$ can become large,

$$
R \ll \ell \ll l_{0} .
$$

This means that Eqs. (41) and (44) for the scattering probabilities are valid not only for the weak roughness (42), (51), but also for a much stronger high-aperture roughness (58), but only to the extent that one is interested in the transitions to and from the gravitational states with exponentially small wave functions on the rough wall. In this case one can often disregard the correlation exponents $\exp \left[-\left(\beta_{j}-\beta_{j^{\prime}}\right)^{2} r^{2} / 2\right]$ in the expressions for $W$ and make the rest of the calculations as if the roughness were weak. Physically the disappearance of these exponents means that the changes of momenta in scattering are now unrestricted. However, the moment the transitions between the higher states come into play, these equations loose their accuracy.

On the other hand, the large amplitude of roughness always leads to a completely different picture than the weak roughness. Note that if we are interested in identification of the gravitational states when the distance between the walls $H$ is comparable to the level size $l_{0}, H \gtrsim l_{0}$, the condition $\ell \ll l_{0}$ is a necessary condition for the existence of the gravitational states. Therefore, we have to assume that this condition holds and that the only important type of strong roughness is the one given by Eq. (58). In the case of highamplitude roughness with $\ell \gg l_{0}$, Eq. (35) still holds for the gravitational states and the structure of Eqs. (41), (44) remains the same, though, instead of the correlation function of surface inhomogeneities $\zeta\left(\pi_{j}-\pi_{j^{\prime}}\right)$, the expressions for $W$ contain the summation over the higher-order correlators. However, as it was explained above, we are not interested in this situation. What is more, under these conditions the state energies experience such large roughness-driven changes that the classification of states on the basis of an ideal well loses all meaning.

For practical purposes, the results of this section mean that the our theory is still valid for a relatively strong roughness (58), but only in application to the lowest gravitational states and not to the square-well states. Since in this paper we were interested mostly in the direct transitions from the gravitational states into continuum, these results still hold for $1 \gg \eta \gg r$ and the results in Fig. 11 are still within the applicability of the theory and the step-wise depletion of neutrons can be observed.

\section{RELAXATION TIMES}

\section{A. Direct absorption vs protracted diffusion between the states}

There are two ways in which the neutrons can be absorbed by the plates (transit into the continuous spectrum) as a result of scattering-driven interstate transitions. The first way is a relatively protracted diffusion between the states which results, eventually, in neutron getting into the continuum. The second way is by direct transitions from the initial state into the continuum without involving any intermediate states. For the latter method to dominate, the probabilities of direct transitions into continuum should dominate over transitions between individual discrete states. Obviously, this direct process has an undeniable theoretical appeal since in this case we do not need to solve the set of $120 h \times 120 h$ transport equations (25) and only calculate the transition times for direct transitions into continuum $\tau_{j}^{(0)}$.

However, as we will see below, following this route has important advantages for experiment as well. If the direct transitions dominate, the results will be the least sensitive to the correlation characteristics of the surface roughness (both to the correlation parameters and to the shape of the correlation function) and to the details of the initial distribution of neutrons over the energy levels. Then the interpretation of experimental data should be more reliable and unambiguous than is the case of protracted interstate diffusion.

We will start by delineating conditions under which the direct transitions into the continuum from all discrete states, including the lowest, are possible and dominant. The next step is the calculation of the corresponding transition rates which will immediately lead us to the results for the neutron count.

The most relevant time scale is the time of flight,

$$
t=L_{x} / V_{0} \sim 2 \times 10^{-2} \mathrm{~s} .
$$

Numerically, the scale of the relaxation times in (26) is given, according to Eqs. (44)-(48), by the coefficient $\tau_{0}$ (45) in the scattering probabilities $W$,

$$
\tau_{0}^{-1}=\frac{\sqrt{2 \pi}}{4 m^{2}} \frac{\hbar^{2}}{l_{0}^{3} v_{0}} \eta^{2} \sim 1.15 \times 10^{3} \eta^{2} \mathrm{~s}^{-1},
$$

where $\eta=\ell / l_{0}$ is the dimensionless amplitude of the surface corrugation, and $t / \tau_{0} \sim 23 \eta^{2} / \beta$. Since $l_{0} \sim 5.871 \mu \mathrm{m}$ and the 
spacing between the walls in experiment does not exceed $50 \mu \mathrm{m}$, the reasonable limits on the amplitude of inhomogeneities are $10^{-3} \leq \eta=\ell / l_{0} \leq 0.1$, and

$$
2.3 \times 10^{-5} \lesssim t / \tau_{0} \lesssim 0.23
$$

[in existing experiments, $0.03 \lesssim \eta \lesssim 0.1$ and the lower limit in the inequality (61) is about 0.02$]$.

The full relaxation times differ from $\tau_{0}^{-1}$ by the dimensionless factors $w$. To simplify the problem, we have to follow the largest of these $w$. Note, that according to the above expressions for $W$ [see, e.g., Eq. (47)] the most probable transitions are the transitions into the highest allowed (by the correlation exponent) states. Therefore, the direct transitions into the continuous spectrum, when allowed, have the shortest transition times. What is more, the corresponding inverse times $\tau_{j}^{(0)-1}$ contain a summation over all the accessible states in the continuum and could become noticeably shorter than the transition times between the individual discrete states.

There are two types of factors entering the dimensionless probabilities $w$ : the correlation exponents with the negative indices $-\left(\beta_{j}-\beta_{j^{\prime}}\right)^{2} r^{2} / 2$, which can only decrease $w$, and the factors related to the values of the wave functions on the walls. The correlation exponents, in general, encourage the transitions with the smallest change in momentum, i.e., the transitions between the nearby states, unless, of course, the correlation length $r$ is small (see below) and it does not matter. The coefficients originating from the wave functions on the wall, on the other hand, favor the direct transitions into (and from) the highest states [factors $j^{\prime 2} / h^{3}$ in Eqs. (47) and (48)]. The balance of these opposing factors determines whether the attenuation of the gravitational states occurs via the gradual diffusion between the states or via direct transitions into the highest states.

The possibility of the transitions is determined by the momentum transfer in the exponent of the correlation function,

$$
\left(\beta_{j}-\beta_{j^{\prime}}\right)^{2} r^{2} / 2=\left(\sqrt{e-\lambda_{j}}-\sqrt{e-\lambda_{j^{\prime}}}\right)^{2} r^{2} / 2 .
$$

This means that the direct transitions from the lowest states into the continuous spectrum (and all transition between the discrete states) are not suppressed if

$$
(\sqrt{1 / \chi}-\sqrt{1 / \chi-1})^{2} u_{c} r^{2} / 2 \lesssim 1
$$

$\left(\chi \equiv u_{c} / e\right)$ or, using the numeric value for $u_{c}$,

$$
\begin{aligned}
r \lesssim & 3.8 \times 10^{-3} F_{0}(\chi), \quad R \lesssim 2.2270 \\
& \times 10^{-2} F_{0}(\chi) \mu \mathrm{m}, \quad F_{0}(x)=\sqrt{x} /(1-\sqrt{1-x}),
\end{aligned}
$$

where the function $F_{0}(\chi)$ is given in Fig. 2.

This condition is not overly restrictive. Unless the overall velocity of the beam is close to the absorption threshold, i.e., unless $\chi=u_{c} / e$ is close to 1 , the condition (64) can easily be satisfied. Essentially, condition (64) determines whether the particles, which are initially in the lower discrete states, can disappear as a result of direct transitions into continuous spectrum (or into short-lived upper states).

Since the transitions between the lower states are much less likely than the transitions between the lower and higher states and between the higher states, we can use expressions

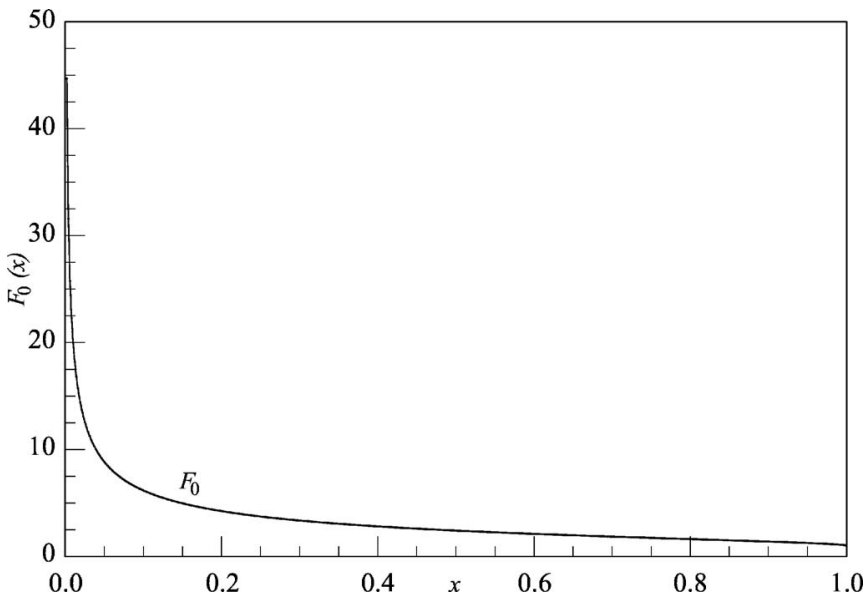

FIG. 2. Function $F_{0}(x)$, Eq. (64).

(48) for all $\tau_{j j^{\prime}}^{-1}$ for the gravitational states and Eq. (47) for all square-well states.

\section{B. Transitions from the square-well states into continuous spectrum (direct absorption)}

We should start by estimating the times $\tau_{j}^{(0)}$ for transitions into continuous spectrum (50): if these times are short, at least for some discrete states $j, t / \tau_{j}^{(0)} \gg 1$, this will help in evaluating the relaxation times for all other states as well. First, since $u_{c}$ is large, one can replace $\cos ^{2}\left(h \sqrt{\lambda+u_{c}}\right)$ in the denominator of Eq. (50) by $1 / 2$,

$$
\begin{aligned}
\frac{1}{\tau_{j}^{(0)}}= & \frac{u_{c}^{2} l_{0} r \psi_{j}^{2}(h)}{\pi \tau_{0} \beta_{j}} \int_{0}^{e-u_{c}} \frac{d \lambda}{\sqrt{\lambda+u_{c}}} \\
& \times \frac{\exp \left[-\left(\sqrt{e-\lambda_{j}}-\sqrt{e-u_{c}-\lambda}\right)^{2} r^{2} / 2\right]}{1+\left(1+u_{c} / \lambda\right) / 2} .
\end{aligned}
$$

Since $\lambda_{j}<u_{c}<e$, the index in the exponent changes from $\left(\sqrt{e-\lambda_{j}}-\sqrt{e-u_{c}}\right)^{2} r^{2} / 2$ to $\left(e-\lambda_{j}\right) r^{2} / 2>\left(e-u_{c}\right) r^{2} / 2 \sim 10^{5} r^{2}$. If $r$ is small, $r \leqq 10^{-3}(R \leqq 5 \mathrm{~nm})$, as suggested in the previous section, the index in the exponent is always small and the correlation exponent can be disregarded,

$$
\begin{aligned}
& \frac{1}{\tau_{j}^{(0)}}=\frac{u_{c}^{2} l_{0} r \psi_{j}^{2}(h)}{\pi \tau_{0} \beta_{j}} \int_{0}^{e-u_{c}} \frac{d \lambda}{\sqrt{\lambda+u_{c}}} \frac{2}{3+u_{c} / \lambda} \\
&=\frac{u_{c}^{5 / 2} l_{0} r \psi_{j}^{2}(h)}{\pi \tau_{0} \beta_{j}} 2 \int_{0}^{1 / \chi-1} d z \frac{z}{3 z+1} \frac{1}{\sqrt{1+z}} \\
& \approx 10 \frac{u_{c}^{5 / 2} l_{0} r \psi_{j}^{2}(h)}{\pi \tau_{0} \beta_{j}} F_{1}(\chi), \\
& F_{1}(x) \approx 0.11\left[1.2 / \sqrt{x}-1.76+0.245 \ln \left(\frac{6}{3-2.45 \sqrt{x}}-1\right)\right] .
\end{aligned}
$$

The function $F_{1}(x)$, is plotted in Fig. 3 .

For the estimates, the function $F_{1}(\chi)$ is mostly irrelevant except for $e \rightarrow u_{c}(\chi \rightarrow 1)$. According to Eq. (17), for deep square-well levels, 


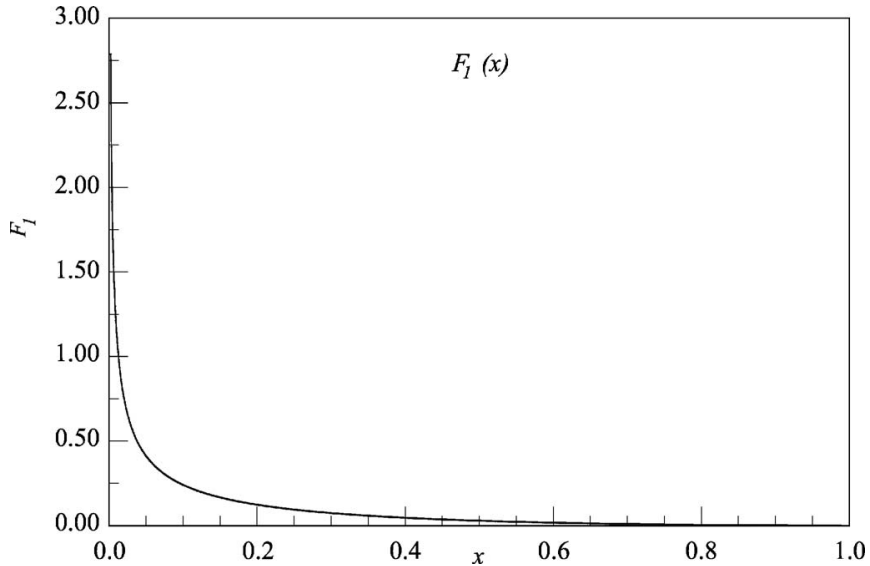

FIG. 3. Function $F_{1}(x)$, Eq. (66).

$$
\frac{1}{\tau_{j}^{(0)}} \sim \frac{4.6 \times 10^{13} r}{\beta_{j} \tau_{0}} \frac{2 \bar{\lambda}_{j}}{h u_{c}} F_{1}(\chi) .
$$

[For higher levels, one should use for $\psi_{j}^{2}(h)$ a more accurate Eq. (15).]

Taking into account Eqs. (59) and (60) for the time of flight $t$ and characteristic time $\tau_{0}$, it is convenient to represent the ratio of the time of flight to the absorption time as

$$
\frac{t}{\tau_{j}^{(0)}} \sim 10^{4} \eta^{2} \frac{\left(10^{3} r\right)}{h\left(10^{-3} \beta_{j}\right)}\left(10^{5} \lambda_{j} / u_{c}\right) F_{1}(\chi) .
$$

Since even for the lowest square-well states $\lambda_{j} / u_{c}>10^{-5}$, this means that neutrons from all the square-well states, even for the lowest ones, disappear directly into the continuous spectrum during the time of flight at any reasonable amplitude of roughness and small lateral size of inhomogeneities as long as the correlation radius of surface roughness $r$ is sufficiently small and the absorption threshold is not close to the overall kinetic energy (the ratio $\chi=u_{c} / e$ is not close to 1).

At larger size of inhomogeneities, the restrictions on the momentum transfer become more important and the integral in Eq. (65) should be evaluated more carefully. For the lower states $\lambda_{j} \ll e$, the absorption time is given by Eq. (66) in which the integral $F_{1}(\chi)$ should be replaced by the function $F\left(\chi, \sqrt{u_{c}} r\right)$ :

$$
\begin{gathered}
\frac{1}{\tau_{j}^{(0)}}=10 \frac{u_{c}^{5 / 2} l_{0} r \psi_{j}^{2}(h)}{\pi \tau_{0} \beta_{j}} F\left(\chi, \sqrt{u_{c}} r\right), \\
F(x, y)=0.2 \int_{0}^{1 / x-1} d z \frac{z}{3 z+1} \frac{1}{\sqrt{1+z}} \\
\quad \times \exp \left[-(\sqrt{1 / x}-\sqrt{1 / x-1-z})^{2} y^{2} / 2\right] .
\end{gathered}
$$

The plots of the function $F\left(\chi, \sqrt{u_{c}} r\right)$ at two values of $r, r$ $=0.01,0.001$ are given in the Fig. 4. The same function $F\left(\chi, \sqrt{u_{c}} r\right)$ is plotted as a function of $r$ in Fig. 5 at $\chi=0.15$, $0.3,0.5$. As can be seen in the plots, choosing proper values for the correlation radius of inhomogeneities $r$ and the ratio of the threshold energy to the overall kinetic energy is crucially important for having effective absorption.

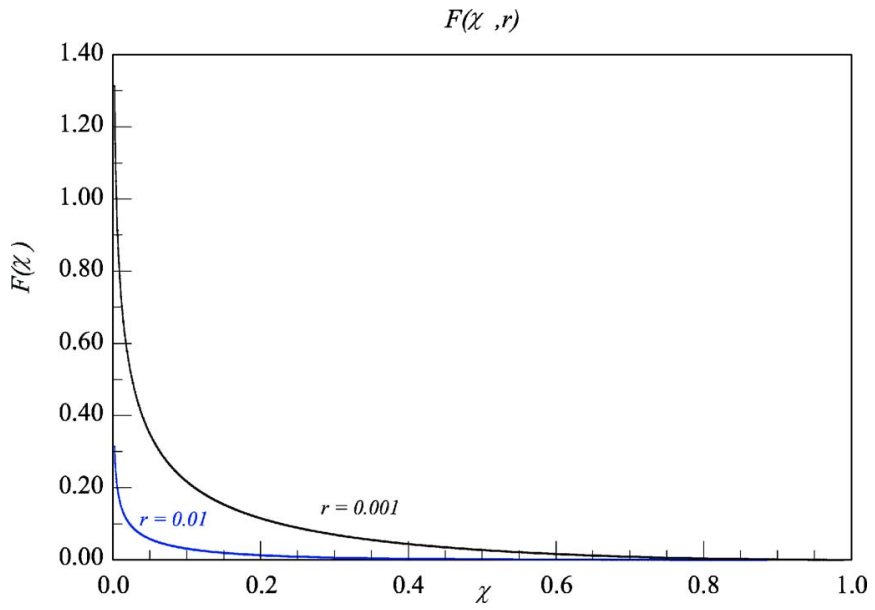

FIG. 4. (Color online) Function $F\left(\chi, \sqrt{u_{c}} r\right)$, Eq. (69), at two values of $r, r=0.01,0.001$.

The function $F\left(\chi, \sqrt{u_{c}} r\right)$ decreases rapidly with increasing $\chi$ and even more rapidly with increasing $r$. Since $\sqrt{u_{c}} \sim 370$ and the integrand depends exponentially on $\sqrt{u_{c}} r$, the dependence of $F\left(\chi, \sqrt{u_{c}} r\right)$ on $r$ is very steep: when $r>0.03$ the momentum transfer restrictions are so severe that the direct transitions from the lower levels to the continuous spectrum are suppressed by orders of magnitude unless $\chi$ is very small (in existing experiment, $\chi \geqq 0.15$ ). For example, when $r=0.01$ the function $F\left(\chi, \sqrt{u_{c} r}\right)$ reaches $10^{-2}$ only at $\chi$ $\simeq 0.26$.

With the same numbers as in Eq. (68), the ratio of the time of flight to the time of direct transitions into the continuous spectrum is

$$
\frac{t}{\tau_{j}^{(0)}} \sim 10^{4} \eta^{2} \frac{\left(10^{3} r\right)}{h\left(10^{-3} \beta_{j}\right)}\left(10^{5} \lambda_{j} / u_{c}\right) F\left(\chi, \sqrt{u_{c}} r\right) .
$$

\section{Direct transitions from the gravitational states into the continuous spectrum}

Probabilities of direct transitions from the gravitational states into continuous spectrum are given by the same Eq.

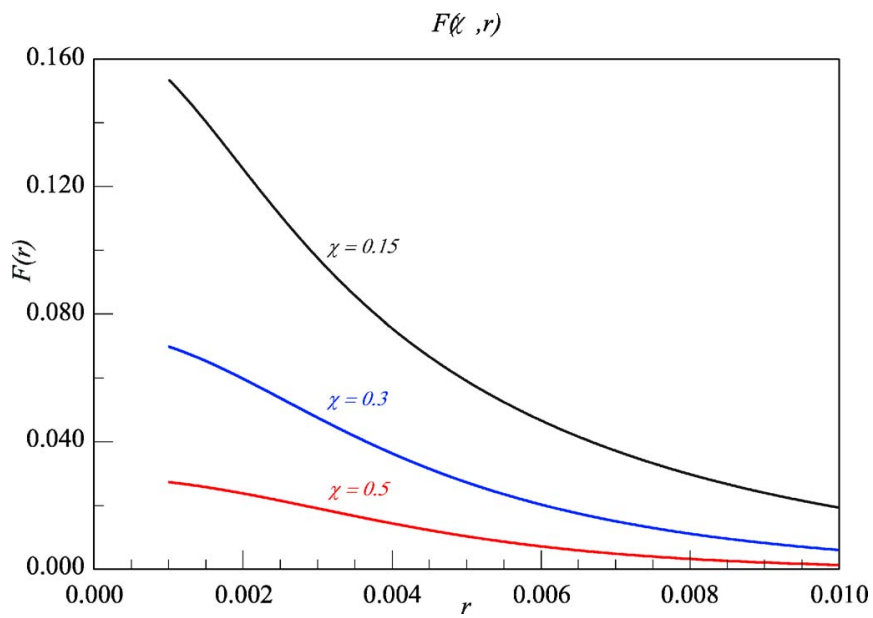

FIG. 5. (Color online) Function $F\left(\chi, \sqrt{u_{c}} r\right)$, Eq. (69), at three values of $\chi, \chi=0.15,0.3,0.5$. 


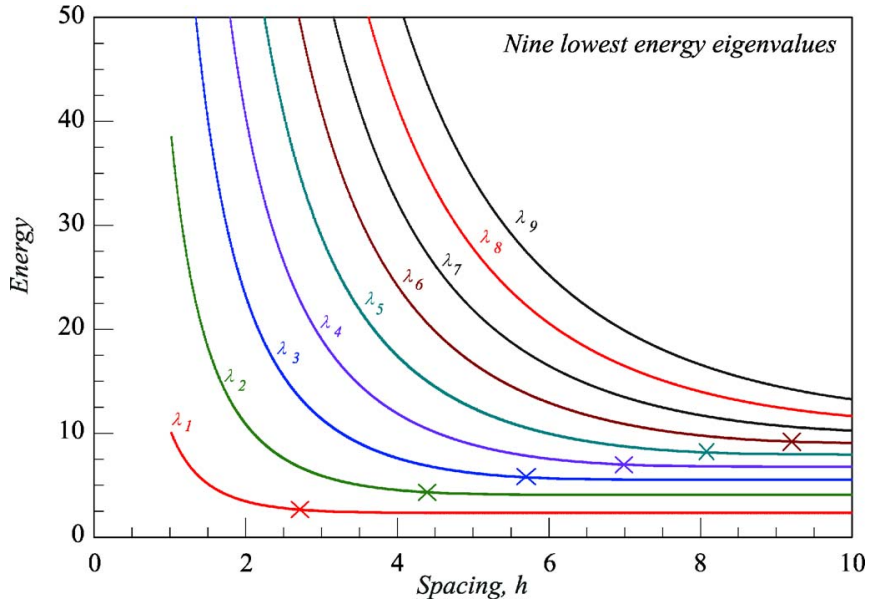

FIG. 6. (Color online) Nine lowest energy levels $\lambda_{i}$ as a function of the spacing between the walls $h$. The stars mark the positions where the size of the energy of the level becomes equal to $m g H$ (in dimensionless variables, $\lambda_{i}=h$ ) and the level shifts to the squarewell domain.

(65), but with different values of $\psi_{j}(h)$, Eq. (12) instead of Eq. (15). Since the integral remains the same, the only substantial change is the replacement of $\psi(h)$ in the right-hand side of Eq. (69) by $B_{j}(12)$,

$$
\begin{gathered}
\frac{t}{\tau_{j}^{(0)}} \sim 10^{4} \eta^{2} \frac{\left(10^{3} r\right)}{10^{-3} \beta_{j}} b_{j} F\left(\chi, \sqrt{u_{c}} r\right), \\
b_{j} \equiv 10^{5} l_{0} B_{j}^{2} / 2=10^{5} a_{j}\left[\mathrm{Ai}^{\prime}\left(h-\bar{\lambda}_{j}\right)-\bar{S}_{j} \mathrm{Bi}^{\prime}\left(h-\bar{\lambda}_{j}\right)\right]^{2} / 2 u_{c} \\
\simeq 0.3 a_{j}\left[\mathrm{Ai}^{\prime}\left(h-\bar{\lambda}_{j}\right)-\bar{S}_{j} \mathrm{Bi}^{\prime}\left(h-\bar{\lambda}_{j}\right)\right]^{2}
\end{gathered}
$$

where $a_{j}$ is given by Eq. (8),

$$
\begin{aligned}
a_{j}= & \left\{\left[\mathrm{Ai}^{\prime}\left(-\bar{\lambda}_{j}\right)-S \mathrm{Bi}^{\prime}\left(-\bar{\lambda}_{j}\right)\right]^{2}\right. \\
& \left.-\left[\mathrm{Ai}^{\prime}\left(h-\bar{\lambda}_{j}\right)-\bar{S} \mathrm{Bi}^{\prime}\left(h-\bar{\lambda}_{j}\right)\right]^{2}\right\}^{-1} .
\end{aligned}
$$

This means that the neutrons in the gravitational states disappear in the time of flight unless the coefficient $a_{j}\left[\mathrm{Ai}^{\prime}\left(h-\bar{\lambda}_{j}\right)-\bar{S}_{j} \mathrm{Bi}^{\prime}\left(h-\bar{\lambda}_{j}\right)\right]^{2}$ and/or the function $F\left(\chi, \sqrt{u_{c}} r\right)$ are very small. This is so when the distance between the walls $h$ significantly exceeds $\bar{\lambda}_{j}$ and the wave function on the wall and, therefore, $b_{j}$ are exponentially small. To evaluate this effect we need to calculate numerically the eigenvalues $\bar{\lambda}_{j}(h)$, Eq. (7), and the coefficients $b_{j}$, Eq. (71).

The dependence of the nine lowest (gravitational) energy levels on the spacing is presented in Fig. 6. The crosses on the curves mark the points where the "size" of the level becomes equal to the distance between the walls (in our notations $\lambda=h$ ) and the wave functions start "touching" the walls or, in other words, the levels move from the gravitational to the square well domain.

The coefficients $b_{j}(h)$, Eq. (71), are presented in Figs. 7 and 8 in two different scales. As we can see, the curves are extremely steep. This means that when scanning the distance between the walls $h$ one encounters the critical values $h_{j}$ at

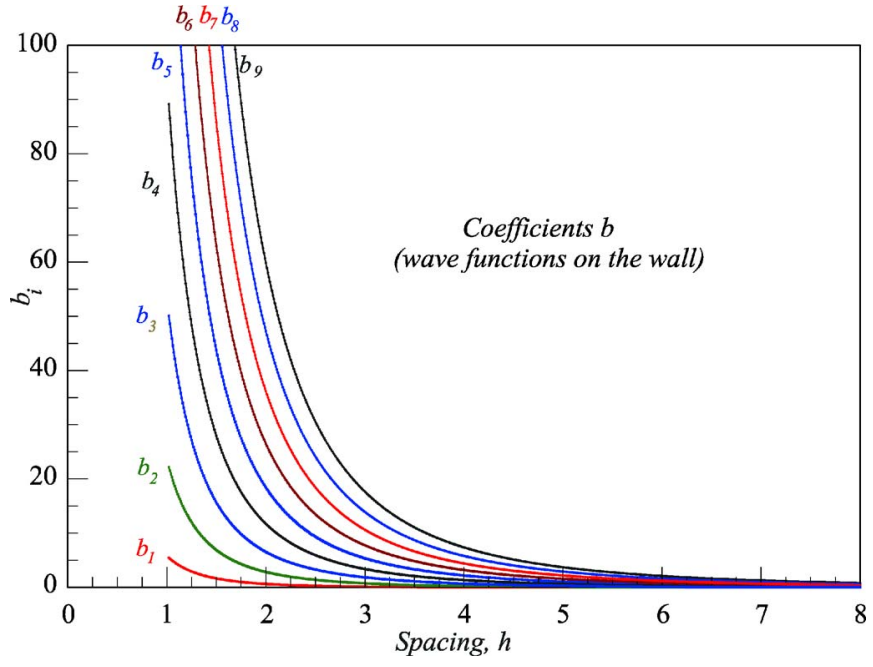

FIG. 7. (Color online) The coefficients $b_{j}(h)$, Eq. (71), which determine the square of the wave function on the surface of the scatterer, $B_{j}^{2}$.

which $t / \tau_{j}^{(0)}$ changes from very small to very large values. According to numerical data, $b_{j}(h)$ changes between $10^{-4}$ and $10^{-2}$ when $5.87>h>4.13$ for $b_{1}, 6.64>h>5.76$ for $b_{2}$, $8.62>h>7.12$ for $b_{3}, 10>h>8.34$ for $b_{4}$, etc. The change of $b$ for all these levels from $10^{-4}$ to $10^{-3}$ requires change in $h$ by only 0.2 . Since such change in $h$ is equivalent to the change in the distance between the walls by about $1.1 \mu \mathrm{m}$ and the neutron count depends exponentially on the coefficients $b$, the neutron count should, under proper circumstances, become in experiment a truly stepwise function of the clearance between the walls. However, since the functions $b(h)$ for higher levels overhang over each other, it is rather unlikely if within this observation technique one can resolve more than three or four gravitational levels.

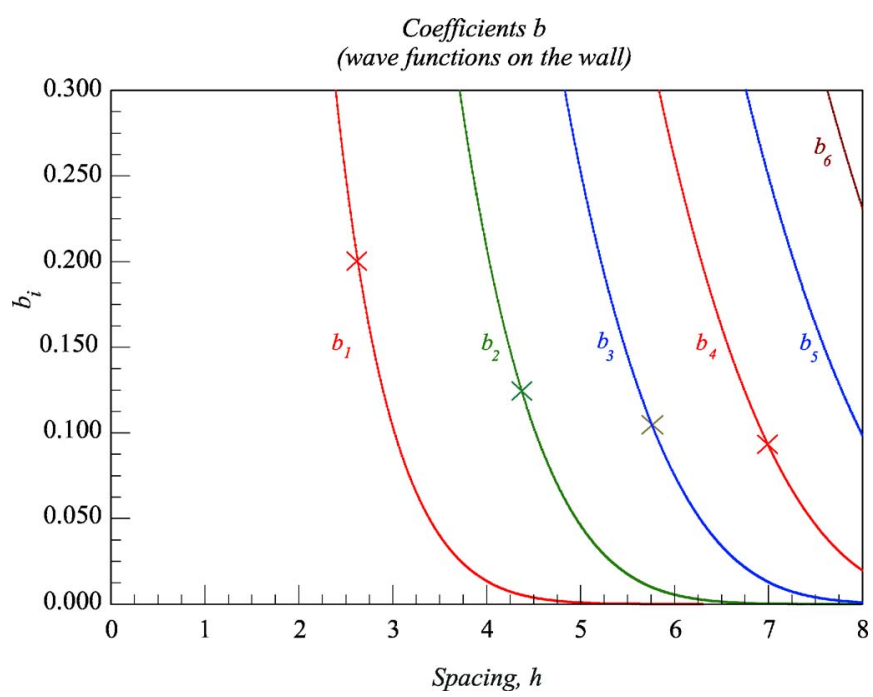

FIG. 8. (Color online) The same as in Fig. 7 but on a smaller scale. The stars on the curves mark the same points as in Fig. 6. 


\section{Neutron count}

As long as the main route for the neutron absorption is the direct transition into continuous spectrum, the neutron count at the exit is

$$
N(t)=\sum N_{j}(0) \exp \left(-t / \tau_{j}^{(0)}\right) .
$$

The count, of course, depends on the initial number of neutrons in each state, $N_{j}(0)$. When the only long-lived states are the few lowest ones with the energies much lower than the overall kinetic energy of particles in the beam and than the scale of the energy dispersion, it is reasonable to assume that the initial populations of these states are the same, $N_{j}(0)$ $=N_{0}$, and

$$
n(h)=N(t) / N_{0}=\sum \exp \left(-t / \tau_{j}^{(0)}\right),
$$

while for each level

$$
n_{j}(h)=N_{j}(t) / N_{j}(0)=\exp \left(-t / \tau_{j}^{(0)}\right)
$$

with $t / \tau_{j}^{(0)}$ is given by Eq. (71). In reality, $t=L / v_{x}$ is fixed and $n$ should be considered not a function of the time of flight $t$, but as a function of the clearance between the walls $h$.

Below we plot the functions $n(h)$ and $n_{j}(h)$ for the overall neutron count and individual level depletions. According to the definition (74), the neutron count from the individual level $n_{j}(h)$ always changes from 1 at large $h$ to 0 at small $h$. The numerical value of the overall neutron count $n(h)$ also provides the information about a number of nondepleted levels: for $n(h)>1$, more than one level is still occupied; for $n(h)>2$, more than two levels are still occupied; for $n(h)>2$ more than three levels are still occupied; etc.

Since the functions $b_{j}(h)$ grow by orders of magnitude when $h$ changes from 6 to 1 , the ratios $t / \tau_{j}^{(0)}$ also change by orders of magnitude and the neutron count $n(h)$ should decrease very steeply. In principle, if the absorption times $\tau_{j}^{(0)}$ for different levels are vastly different, the curve $n(h)$ [Eq. (73)] should become a stepwise function with each step representing the decay of an individual state $n_{j}(b)$, Eq. (74). Unfortunately, this is not what happens.

First, we will plot the function $n(h)$ which describes the steepest achievable depletion of the neutron population, while still remaining within the applicability of the weak roughness theory. In other words, we will plot $n(h)$ which corresponds to the largest combination of coefficients in Eq. (70). Since the weak roughness theory is valid for roughness with the amplitude $\eta$ smaller than the correlation radius of inhomogeneities $r$, the largest $t / \tau_{j}^{(0)}$ with given $r$ is achieved at $\eta=r$. The function $F\left(\chi, \sqrt{u_{c} r}\right)$, on the other hand increases with decreasing $\chi$ though in experiment this parameter does not go below 0.15 . The plot of the function $10^{7} r^{3} F\left(\chi, \sqrt{u_{c}} r\right)$, which enters the ratio $t / \tau_{j}^{(0)}$, Eq. (70), as a coefficient, is presented in Fig. 9 for three values of $\chi$. Clearly, the optimal conditions for experiment are $\chi=0.15, \eta=r=0.015$ when $10^{7} \eta^{2} r F\left(\chi, \sqrt{u_{c}} r\right) \sim 0.23$.

The neutron count (73) with these values of $\chi, \eta, r$ is presented in Fig. 10 as a function of the interwall clearance. The

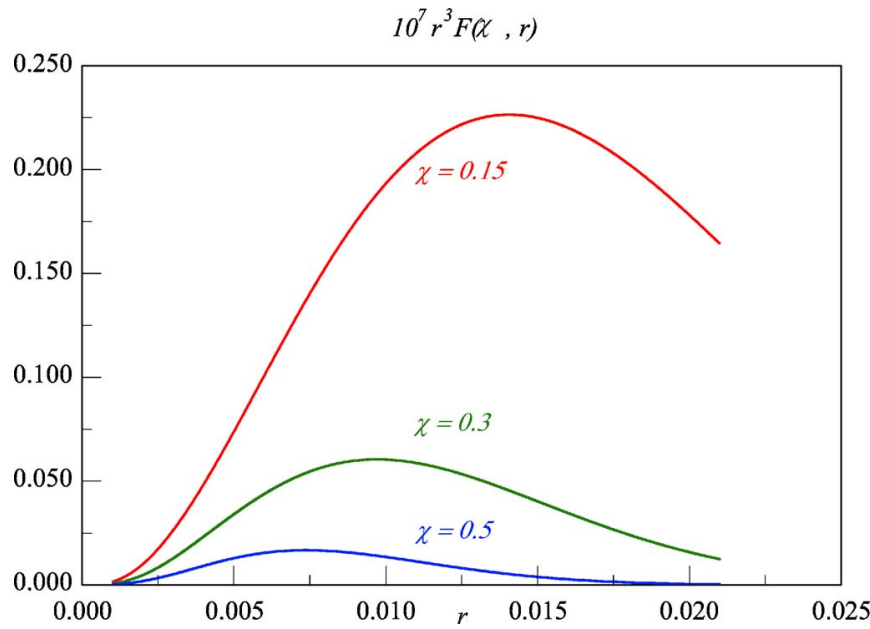

FIG. 9. (Color online) Function $10^{7} r^{3} F\left(\chi, \sqrt{u_{c}} r\right)$ for Eq. (71) at three values of $\chi, \chi=0.15,0.3,0.5$.

same figure also gives the individual depletions of the nine lowest quantum levels (74).

This figure clearly shows that though the depletion is sufficiently steep for an unambiguous demonstration of the quantization of the gravitational states (the total count is steep and goes to zero at $h \neq 0$ ). However, the "overhang" of the coefficients $b_{j}(h)$ (Figs. 7 and 8) is too large for identification of the individual levels: though the individual depletion rates are clearly distinguishable, the overall neutron count is still a featureless function of $h$. This overhang can be compensated only by an increase in other coefficients in Eq. (70). This result means that the use of a scatterer with slight roughness is insufficient, even under the most favorable circumstances, for an observation of a stepwise change in neutron count and, therefore, for an identification of higher states, unless parameter $\chi$ becomes noticeably smaller.

On the other hand, the use of large roughness such as, for example, an increase in the amplitude of wall roughness to

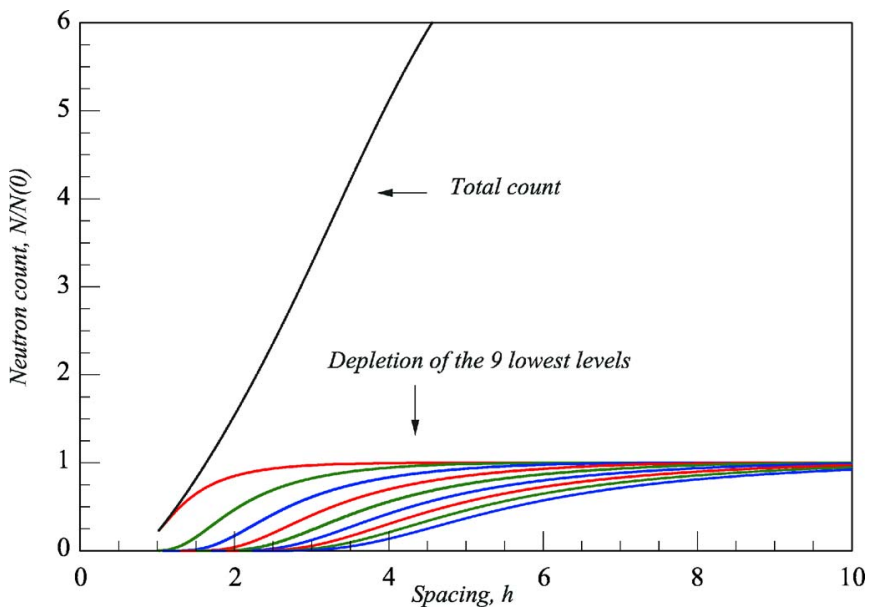

FIG. 10. (Color online) The neutron count $N / N(0)$, Eq. (73), and individual depletions of the nine lowest energy levels, Eq. (74), as a function of the spacing between the walls $h$ at $\chi=0.15, \eta=r$ $=0.01$. 


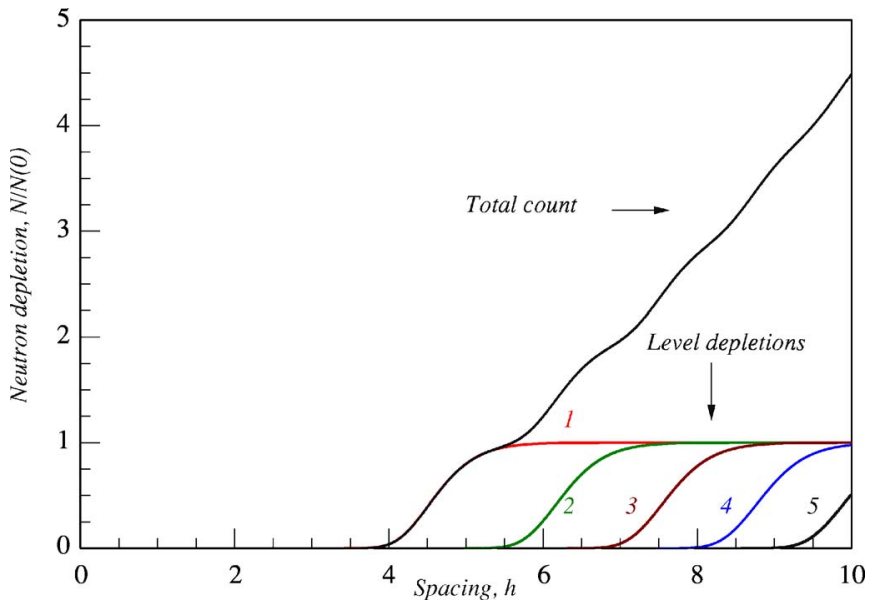

FIG. 11. (Color online) The neutron count $N / N(0)$, Eq. (73), and individual depletions of the lowest energy levels, Eq. (74), as a function of the spacing between the walls $h$ at $\chi=0.15, r=0.015$, $\eta=30 r$. All higher levels are already depleted.

the value $\eta=30 r$, would modify the neutron count in Fig. 10 to the one depicted in Fig. 11 with very clear steps that separate the depletions of the individual levels. Though the parameters themselves $r=0.015, \eta=0.45$ are reasonable from the point of view of experiment, the large ratio $\eta / r=30$ is out of range of the slight roughness theory. However, the theory can be extended, as is explained in Sec. IV, to a lowamplitude high-aperture roughness and can cover the situation plotted in Fig. 11.

Though the existing experiment does not produce as unambiguous picture as in Fig. 11, the similarities indicate that in experiments the roughness was not small. Additional information on the evolution of neutron count as a function of $\eta / r$ can be found in Fig. 14.

\section{E. Alternative geometries}

Similar experiments have been performed in alternative geometries with, for example, a mirror on the top and the rough scatterer on the bottom. The theoretical description of the change in neutron population with time remains the same as above with the only difference that all transition probabilities are now proportional to $|\psi(0)|^{2}$ instead of $|\psi(H)|^{2}$. For upper states this does not make any difference since the wave functions are symmetric and $|\psi(0)|^{2}=|\psi(H)|^{2}$. This is not so for the gravitational states (12) for which the wave functions on the top and on the bottom plates can differ by orders of magnitude.

The ratio of the time of flight $t$ to the relaxation times $\tau_{j}^{(0)}$ for the gravitational states in this alternative geometry is given, instead of Eq. (12), by the following expression:

$$
\begin{gathered}
\frac{t}{\tau_{j}^{(0)}} \sim 10^{4} \eta^{2} \frac{\left(10^{3} r\right)}{10^{-3} \beta_{j}} c_{j} F\left(\chi, \sqrt{u_{c} r}\right), \\
c_{j}=b_{j}\left(\frac{\mathrm{Ai}^{\prime}(-\bar{\lambda})-\bar{S} \mathrm{Bi}^{\prime}(-\bar{\lambda})}{\mathrm{Ai}^{\prime}\left(h-\bar{\lambda}_{j}\right)-\bar{S}_{j} \mathrm{Bi}^{\prime}\left(h-\bar{\lambda}_{j}\right)}\right)^{2} .
\end{gathered}
$$

As expected, the coefficients $c_{j}$ are larger than the coefficients $b_{j}$, especially when the spacing between the walls is

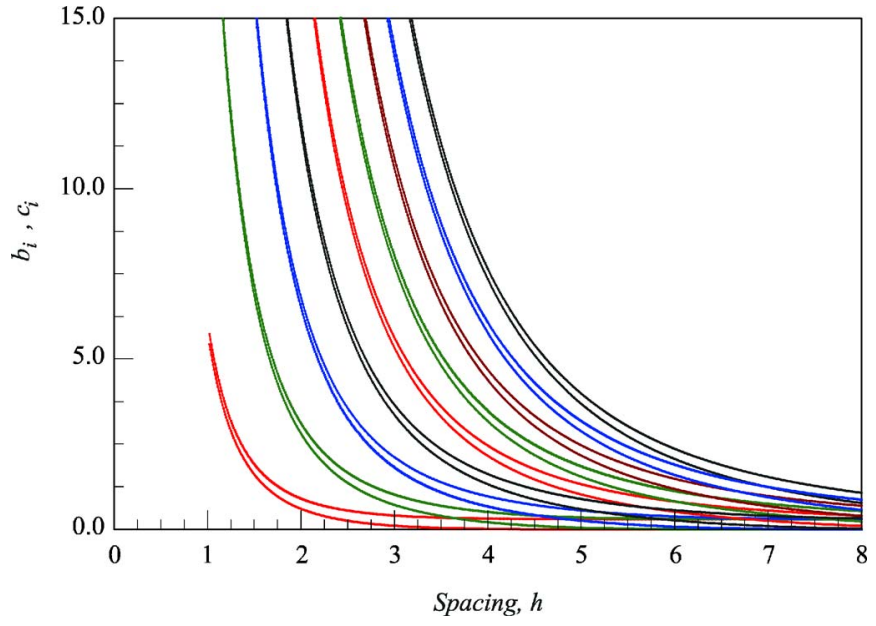

FIG. 12. (Color online) Pairs of coefficients $b_{j}(h)$ (lower curves) and $c_{j}(h)$ (upper curves), Eqs. (71), (75), which describe the square of the wave function on the surfaces $s=h$ and $s=0$ for the lowest nine states. Merging of these coefficients at small spacing between the walls $h$ correspond to the transformation of the gravitational states into the symmetric square-well states.

large. The difference between these coefficients disappear when the spacing decreases and the states become more symmetric as it is clearly seen in Figs. 12 and 13.

When the coefficients $c_{j}$ and $b_{j}$ are comparable, the corresponding depletion rates for both geometries are also of the same order of magnitude. Under the same conditions as in Fig. 9, though the depletion rates for the first level in direct and inverse geometries are different because of a noticeable difference in $c_{1}$ and $b_{1}$, the depletion rates that include nine levels do not differ much between themselves (Fig. 14; the pair of curves marked $d-1$ and $i-1$ ).

However, with an increase in coefficient (75), the difference in depletion rates between "direct" and "inverse" geometries, which is due to the differences between $c_{j}$ and $b_{j}$,

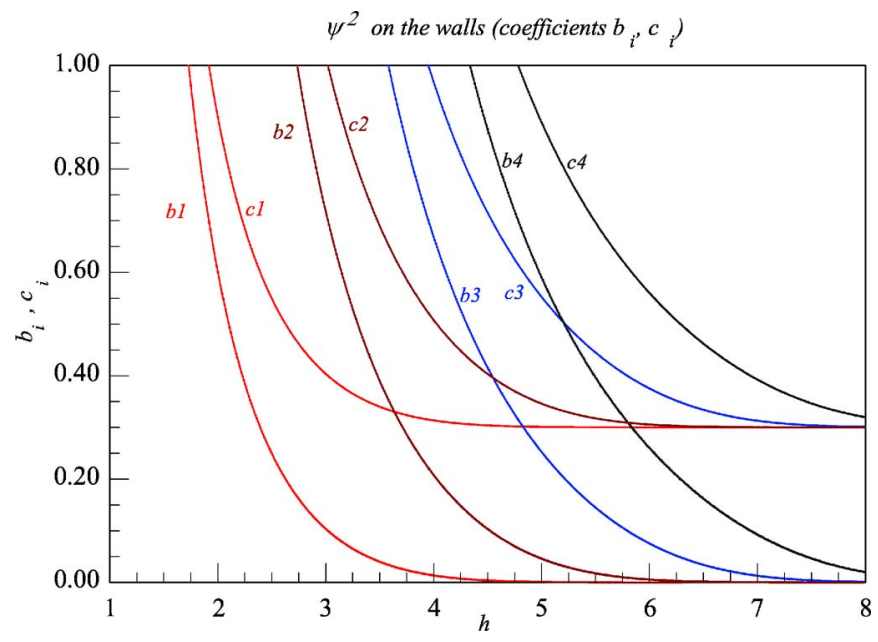

FIG. 13. (Color online) The same coefficients $b_{j}(h)$ and $c_{j}(h)$, Eqs. (71), (75), for the lowest four levels as in Fig. 12, but on a smaller scale. Merging of these coefficients at small spacing between the walls $h$ indicates the transformation of the gravitational states into the symmetric square-well states. 


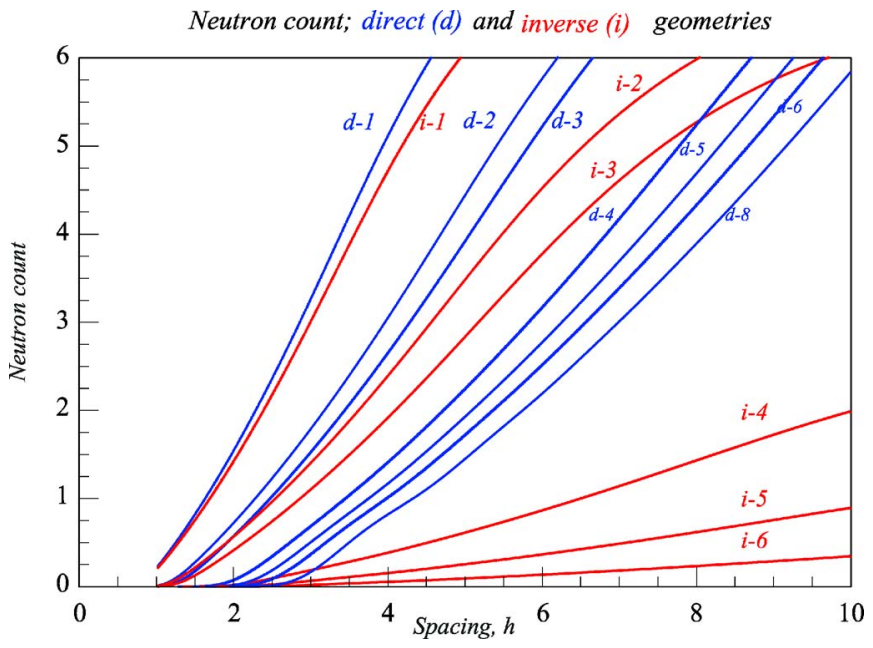

FIG. 14. (Color online) The depletion rates for direct ( $d$; the scatterer is on the top) and inverse ( $i$; the scatterer is on the bottom) geometries for $\chi=0.15, r=0.015$. The curves are marked by the ratio $\eta / r=1,2,3,4,5,6,8$ and by the geometry $d$ or $i$.

becomes more and more pronounced. Figure 14 contains several more pairs of curves for direct and inverse geometries which correspond to the amplitude of roughness equal to $\eta / r=2,3,4,5,6,8$ (the curves are marked accordingly). For even larger roughness, such as in Fig. 11, the depletion rate for the inverse geometry is so fast (the scale is $10^{-14}$ ) that the curves for direct and inverse geometries cannot be plotted in the same figure; even for $\eta=8 r$ the numbers for inverse geometry are already too small. Note that when the spacing between the walls becomes small and the energy levels go sufficiently high up into the square-well domain, the pairs of curves that describe the depletion rates in direct and inverse geometries merge between themselves.

Figure 14 illustrates the evolution of neutron count with increasing aperture of roughness $\eta / r$. According to this figure, the increase of the ratio $\eta / r$ from 1 to 8 is still insufficient to produce a stepwise plot of the neutron depletion with well-pronounced steps as in Fig. 11. However, at $\eta=8 r$ there are already some signs of the appearance of the first step on the depletion curve.

\section{PERSPECTIVES AND CONCLUSIONS}

\section{A. What should we expect from strong-roughness experiments?}

On the face of it, the case for shifting experiments toward strong roughness is clear: the curves should become sharper and the chances of identifying the individual gravitational states higher. However, as usual, there are pitfalls.

The strong roughness can come in two flavors: largeamplitude roughness, comparable to the spacing between the walls or to the size of the gravitational levels, and roughness with small lateral size of inhomogeneities in comparison with their amplitude (high-aperture roughness). The use of the former is rather dangerous. The main problem is that the distance between the walls becomes an ill-defined variable and, because all energy parameters are very sensitive to this spacing, the energies extracted from such experiments are unreliable. Therefore, though the sharpness of the curves improves, the curves, by themselves, can be used for nothing more than qualitative identification of the individual states. The only exception might be the so-called adiabatic roughness [15] in which the profile of the walls changes so slowly that the wave functions can adjust to the local shape of the walls. Still, even in this case the uncertainty in energies is large making the quantitative results useless.

The low-amplitude high-aperture roughness, on the other hand, can both improve the resolution for the lowest states and allow one to extract useful data on the interaction between neutrons and solid surfaces. This can be achieved only when the dominant depletion regime is the direct scatteringdriven absorption of neutrons. The moment the intermediate square-well states become involved, the accuracy of data extracted from experiments with high-aperture walls is lost.

\section{B. Angular resolution}

Another way of increasing the sensitivity of experiment to individual levels is to decrease the parameter $\chi=U_{c} / E$ which describes the ratio of the absorption threshold to the overall kinetic energy of particles: obviously, the smaller is this ratio, the higher are the chances of a particle to get absorbed after scattering. Mathematically this effect is described by a steep function $F\left(\chi, \sqrt{u_{c}} r\right)$, Eq. (69), in Fig. 4.

The experimental control over the value of $\chi$ could be limited. However, there might be an easier alternative to lowering $\chi$. In experiment, and, as a result, in the theory above, very little attention has been paid to the third spatial direction, $y$. What we were looking at was the scattering-driven change of direction of particles from the beam direction $x$ to the vertical direction $z$ which could provide the particle with sufficient kinetic energy $m v_{z}^{2} / 2$ to overcome the absorption threshold $U_{c}$. Obviously, as a result of scattering the particle acquires not only the vertical velocity $v_{z}$, but also the component of velocity $v_{y}$ in the third spatial direction. Though this component of velocity does not affect the chances of the particle to be absorbed by one of the horizontal walls, the particle can, nevertheless, escape the exit counter if the velocity $v_{y}$ and, therefore, the deviation from the initial trajectory, are large enough. On the other hand, the exit neutron counter cannot distinguish whether the neutrons have escaped as a result of absorption or because of angular dispersion of the beam in the $y$ direction. Therefore, instead of decreasing the parameter $\chi$, one can achieve the same effect simply by decreasing the size of the counter in the $y$ direction. Of course, the price of using the low-angle counter will be an overall drop in the number of detected neutrons. Therefore, following this route might require an increase in beam intensity.

From a theoretical standpoint, the use of the angular resolution requires taking into account the scattering in $y$ direction instead of averaging in Eq. (21). Since we have the full 2D expressions for the scattering probabilities $W$, this will lead just to computational complications rather than to conceptual difficulties. For a wide-aperture counter this extension would hardly change the nature of the results. However, for narrower counters, as it is explained above, this might 
turn out to be important. Essentially, we have to introduce a new angular counting threshold and find the proper replacement for the function $F\left(\chi, \sqrt{u_{c}} r\right)$. The structure of the calculations will remain the same especially if we limit ourselves, at least in the beginning, to the calculation of direct highangle scattering processes rather than looking at protracted diffusion of particles over the angles.

\section{Contribution from the intermediate states}

In this paper we investigated only the situation when the direct scattering into the continuum dominates the neutron depletion. In principle, one should take into account processes that include the indirect absorption processes that involve the intermediate-energy states.

When the direct absorption processes are efficient, namely, when $r$ is sufficiently small, adding diffusion over intermediate states should not speed up the absorption. Involving the intermediate states can be beneficial only for some combinations of parameters when the direct processes are suppressed because of a relatively large value of $r$. The absorption via the intermediate states can sometimes compensate for the presence of the correlation exponents with $u_{c} r^{2}$ that slow down the direct absorption processes. On one hand, the probability of scattering-driven transition $\lambda_{i} \rightarrow \lambda_{j}$ is proportional to the square of the wave function on the wall which decreases rapidly with lowering of the state energy $\lambda_{j}$ and, therefore, favors transitions with as large energy increase as possible. On the other hand, the correlation exponent $\exp \left[-\left(\sqrt{e-\lambda_{i}}-\sqrt{e-\lambda_{j}}\right)^{2} r^{2} / 2\right]$ favors transitions into nearby states. The competition of these opposing trends determines at what values of $r$ the intermediate states are important for absorption.

The depletion rates for higher square-well states are always very high. Therefore, at a relatively large value of $r$ there could even be a range of parameters in which the depletion of the gravitational levels via the intermediate state could become much more efficient than the direct transitions over the threshold that the value of the threshold could become irrelevant and the neutron count could lose its dependence on $\chi$.

In this paper we were focused on the search for "optimal" roughness that ensures the steepest neutron depletion when the wave functions start "touching" the walls; under these conditions, the multistage processes via intermediate states does not seem to be necessary. However, the quantitative description of experiment cannot be complete without a more careful look at the role of intermediate states. We plan to address this issue later in a separate presentation.

\section{Short-range forces near the surface}

A fundamental issue is whether it is possible to extract the parameters of the (ultra)short-range forces acting on neutrons near the solid surfaces (and whether such forces exist at all). Our approach to particle diffusion along rough walls, in which the surface roughness is treated in the same way as bulk perturbations, is well suited to answer the question. The short-range forces near the surface can be considered as an- other $\delta$-type perturbation in addition to the roughness-driven perturbation (34). The matrix elements of this additional perturbation are determined by the same equations as the ones that we used for the calculation of the surface-driven transition probabilities. The regular part of these matrix elements determines the shift of the eigenstates $\lambda$ and the values of the wave functions on the walls, $b_{i}, c_{i}$, which are necessary for the calculation of the neutron depletion rates. After such modification the calculation can, in principle, proceed along exactly the same lines as is done above. Whether or not this program will be implemented in the future will be determined by experimental success in observation of a wellpronounced stepwise dependence of the neutron count on the spacing between the walls. If this high resolution is not achieved, the inclusion of the corrections from the shortrange forces will be meaningless.

\section{E. CONCLUSIONS}

In summary, we studied the possibility of detection of the quantized gravitational states of neutrons. We applied our theory of quantum transport along rough surfaces to a neutron beam between flat and rough walls and calculated the roughness-driven transition probabilities between the quantum states. This allowed us to found the depletion rates due to direct absorption processes as a function of the spacing between the walls and parameters of the wall roughness. From the theoretical standpoint, the main achievement is the extension of our theory to systems with strong, high-aperture roughness and the application of the theory to systems with absorbing, leaky walls.

From the point of view of experimental applications, the focus was on finding the optimal roughness parameters that ensure reliable resolution of several gravitational states. The main stumbling block is the overhang of the wave functions from different states which, in most situations, prevents resolution of the nearby states. Apart from the roughness parameters, the neutron count is also very sensitive to the ratio of the particle kinetic energy to the absorption threshold. The most suitable experimental conditions are those in which the characteristic times $\tau_{j}^{(0)}$ for the direct transitions into the continuous spectrum (absorption) are short in comparison to the time of flight except for the lowest gravitational states for which the probability to find the particle near the scatterer or absorber are exponentially small.

According to our results, the preferable conditions for the observation of the well pronounced gravitational states are the following.

(1) Weak roughness is sufficient for establishing the fact of quantization, but is not sufficient for resolving the individual quantum states. The sharpest experimental results within the weak roughness regime are expected when the amplitude and the correlation radius of weak roughness are $\ell \sim R \sim 0.08 \mu \mathrm{m}$.

(2) The resolution of quantum states requires the use of stronger roughness. The use of low-amplitude highaperture roughness $l_{0}, H \gg \ell>R$ is preferable to high amplitude roughness $\ell>l_{0}$ or $\ell>H$. In the latter case, though the levels can be resolved, it is virtually impossible to extract 
useful quantitative information from the experimental data.

(3) According to our results, resolving two or three lowest states would require $\ell \gtrsim 20 R$ when using lowamplitude high-aperture roughness.

(4) In the case of high-aperture roughness with $\eta \gg r$ one can even neglect the correlation exponents and make the rest of the calculations as if the roughness is weak. Physically the disappearance of these exponents means that the changes of momenta in scattering are now unrestricted and the results are now not very sensitive to the correlation radius.

(5) The threshold velocity for the absorption should be noticeably lower than the overall beam velocity, $\chi=U_{c} / E<1$. This condition, if necessary could be replaced by the condition that the width of the detector in the $y$-direction, $L_{y}$ (or the width of the plates), is considerably smaller than the distance to the detector (length of the plates), $L$. In this case, the absorption energy $U_{c}$ is replaced by the threshold for the disappearance of the neutrons in the $y$ direction, $E_{y} \sim\left(L_{y} / L\right)^{2} E$.

We also formulated several suggestions for future theoretical and experimental work which could help in reliable identification of the gravitational states and use of the acquired information for the study of the short-range forces near solid surfaces.

\section{ACKNOWLEDGMENT}

One of the authors (A.M.) is grateful to the ILL group for the hospitality during his stay in Grenoble and for stimulating discussions.
[1] V. V. Nesvizhevsky et al., Nature (London) 415, 297 (2002); Phys. Rev. D 67, 102002 (2003); Eur. Phys. J. C 40, 479 (2005). For additional bibliography see also http:// lpscwww.in2p3.fr/UCN/NiveauxQ_G/publications/index.html

[2] J. H. Freed, Ann. Phys. (Paris) 10, 901 (1985).

[3] K. Hagiwara et al., (Particle Data Group), Phys. Rev. D 66, 010001 (2002).

[4] V. V. Nesvizhevsky and K. V. Protasov, Class. Quantum Grav. 21, 4557 (2004).

[5] A. Yu. Voronin, H. Abele, S. Baeler, V. V. Nesvizhevsky, A. K. Petoukhov, K. V. Protasov, and A. Westphal, Phys. Rev. D 73, 044029 (2006).

[6] A. E. Meyerovich and A. Stepaniants, Phys. Rev. B 60, 9129 (1999); Phys. Rev. B 58, 13242 (1998).

[7] A. E. Meyerovich and I. V. Ponomarev, Phys. Rev. B 65, 155413 (2002).

[8] J. A. Ogilvy, Theory of Wave Scattering from Random Surfaces
(Adam Hilger, Bristol, 1991).

[9] Handbook of Mathematical Functions, edited by M. Abramowitz and I. A. Stegun (Dover, New York, 1972), p. 446.

[10] Y. Cheng and A. E. Meyerovich, Phys. Rev. B 73, 085404 (2006).

[11] G. Fishman and D. Calecki, Phys. Rev. Lett. 62, 1302 (1989); Phys. Rev. B 43, 11581 (1991).

[12] Z. Tesanovic, M. V. Jaric, and S. Maekawa, Phys. Rev. Lett. 57, 2760 (1986); N. Trivedi and N. W. Ashcroft, Phys. Rev. B 38, 12298 (1988).

[13] A. Stepaniants, D. Sarkisov, and A. E. Meyerovich, J. Low Temp. Phys. 114, 371 (1999).

[14] R. M. Feenstra, D. A. Collins, D. Z. Y. Ting, M. W. Wang, and T. C. McGill, Phys. Rev. Lett. 72, 2749 (1994).

[15] A. Kawabata, J. Phys. Soc. Jpn. 62, 3988 (1993). 